\title{
Retention of Carotenes and Vitamin E, and Physico- chemical Changes Occurring upon Heating Red Palm Olein Using Deep-fat Fryer, Microwave Oven and Conventional Oven
}

\author{
Radhika Loganathan ${ }^{1,2}$, Azmil Haizam Ahmad Tarmizi ${ }^{1}$, Shireene Ratna Vethakkan ${ }^{2}$, and \\ Kim-Tiu Teng ${ }^{1 *}$ \\ ${ }^{1}$ Malaysian Palm Oil Board, Bandar Baru Bangi, 43000 Kajang, Selangor, MALAYSIA \\ ${ }^{2}$ Department of Medicine, Faculty of Medicine, University of Malaya, 50603 Kuala Lumpur, MALAYSIA
}

\begin{abstract}
Red palm olein is known to be high in carotenes and vitamin $E$ (tocols) and possess various nutritional benefits. This study evaluates the effect of prolonged heating using three common cooking techniques i.e. deep-fat fryer, microwave oven and conventional oven, on the profiles of carotenes and tocols as well as the physico-chemical changes occurring in red palm olein when compared to conventional palm olein. Physico-chemical changes in all oils were gauged based on their peroxide, $p$-anisidine and total oxidation values, acidity, and fatty acid composition. Both red palm olein and palm olein were thermally stable based on their lower rate of hydrolytic and oxidative degradations as well as higher tocols retention, which allow the oils to undergo heating up to 3 hours using deep-fat fryer and conventional oven. Nevertheless, red palm olein seemed not suitable for prolonged heating processes considering lower retention of carotenes. Microwave heating also influenced the stability of phytonutrients.
\end{abstract}

Key words: red palm olein, palm olein, heating, oxidative stability, carotenes, vitamin E

\section{Introduction}

Palm olein essentially possess greater resistance towards thermal oxidation compared to other vegetable oils and fats $^{1)}$. Due to its inherent thermal properties coupled with the presence of tocols (tocopherols and tocotrienols), palm olein is widely used for various cooking applications ${ }^{2)}$. Tocols, which act as antioxidant, contribute to the stability of palm olein by acting as free radical quenchers. Tocols can prevent hydroperoxide formation in the chain propagation step, or the decomposition process by inhibiting aldehyde formation ${ }^{3)}$. Polyunsaturated oils are reported least thermal stable due to the presence of double bonds, and therefore prone to decomposition. Saturated and monounsaturated oils possess better heat stability and health profile ${ }^{4)}$. During heat treatment, carotenoids and tocols synergistically protect the oils against excessive thermal degradation ${ }^{5}$. Carotene radicals formed during oxidation are converted back into active carotenes in the presence of tocotrienols ${ }^{5}$. Oxidation induces significant loss of oil quality which subsequently affects the functionality, and nutritional values of fried products. Indeed, this reaction triggers the formation of harmful new compounds including polymers, free fatty acids and other oxidative substanc$\mathrm{es}^{6}$.

Cooking oil is commonly used for daily diet preparation. Carotenoid-rich red palm oil is beneficial as it offers vital antioxidants that enhance the well-being of the general population, and fulfil the daily requirement of fatty acids ${ }^{7}$. However, a dietary habit survey involving 200 pregnant Nigerian women showed high prevalence of vitamin A deficiency as a result of high episode of malaria, anaemia, and subclinical infections, i.e. upper respiratory and urinary tract infection, despite $80 \%$ of them consumed red palm oil for cooking. It is suspected that the cooking methods by these women involve excessive heating which contributed to low vitamin $\mathrm{A}$ viability ${ }^{8}$. A study has been conducted to evaluate the thermal stability of various palm products, i.e. palm oil, single- and double fractionated palm oleins, red palm olein and palm-based shortening upon 80 hours of heating at $180^{\circ} \mathrm{C}^{6)}$. This study evaluates thermal and phyto-

\footnotetext{
*Correspondence to: Kim-Tiu Teng, Nutrition Unit, Product Development and Advisory Services Division, Malaysian Palm Oil Board, Bandar Baru Bangi, 43000 Kajang, Selangor, MALAYSIA

E-mail: kimtiu@mpob.gov.my

Accepted December 26, 2019 (received for review August 21, 2019)

Journal of Oleo Science ISSN 1345-8957 print / ISSN 1347-3352 online

http://www.jstage.jst.go.jp/browse/jos/ http://mc.manusriptcentral.com/jjocs
} 
nutrient stabilities of red palm olein under common cooking techniques, i.e. deep-fat fryer, microwave oven and conventional oven, compared to the performance of palm olein. It is hypothesized that phytonutrient-rich red palm olein would possess better thermal stability compared to palm olein with all 3 cooking techniques. The outcomes of this study would provide a holistic perspective about the nutritional properties and thermal resistance of both oils for domestic cooking.

\section{Materials and Methods \\ 2.1 Materials}

Red palm olein and palm olein were obtained from Carotino Sdn Bhd (Pasir Gudang, Johor) of similar batch of production. Standards for tocopherol and tocotrienol homologues (i.e. alpha, beta, gamma and delta)were purchased from Davos Life Sciences Ptd Ltd (Synapse, Singapore); 2,2,5,7,8-pentamethyl-6-hydroxy-chromane (PMC) and alpha carotene from Wako (Osaka, Japan); beta carotene and butylated hydroxytoluene from Sigma Chemical Co. (St. Louis, USA); and retinyl acetate and fatty acid methyl esters from Supelco Analytical (Bellefonte, USA). Solvents; HPLC gradient grade methanol, ethanol, 1-propanol, 2-propanol, acetonitrile, and $n$-hexane were purchased from Merck (Darmstadt, Germany). All other chemicals were purchased from R\&M Chemical (Essex, UK) or Systerm ChemAR (Shah Alam, Malaysia).

\subsection{Heating techniques}

All oils were subjected to heating for a total of $180 \mathrm{~min}$ using deep-fat fryer, microwave oven and conventional oven. About $50 \mathrm{~g}$ of fresh oil was collected before heating while heated oil was sampled for every 15 min across 3 hours of heating. Heated samples were allowed to cool at $60^{\circ} \mathrm{C}$, flushed with nitrogen and stored at $-40^{\circ} \mathrm{C}$ prior to subsequent analyses. The experiments for each oil and heating techniques were performed in duplicates. The protocols for each heating techniques are as follows:

2.2.1 Deep-fat fryer

The heating experiment was performed using a domestic deep-fat fryer (Cornell CDF-S3503, Klang, Malaysia). The fryer was first filled with $3.5 \mathrm{~L}$ of oil and gradually heated to $170 \pm 10^{\circ} \mathrm{C}$ and remained constant at this value across 3 hours of heating. Oil was not replenished throughout the heating period.

\subsubsection{Microwave oven}

A domestic microwave oven (Panasonic NN-SD577M, Shanghai, China) with the capacity of $27 \mathrm{~L}$ was used to heat the oil. Seventy grams of oil were transferred into a ceramic cup and placed at the centre of turntable rotary plate. The sample was then exposed to microwave wattage of $550 \mathrm{~W}$ across the heating period at $170 \pm 10^{\circ} \mathrm{C}$. After heating treatment, the microwave oven door was opened while a fan was used to circulate ambient air to facilitate cooling.

2.2.3 Conventional oven

An aliquot of $70 \mathrm{~g}$ was weighted into aluminum cupcake foil and kept in an oven (Astar DKL-40, Guangdong, China) at $170 \pm 10^{\circ} \mathrm{C}$ from 0 to $180 \mathrm{~min}$. Cooling procedure was also applied following similar manner as microwave oven.

\subsection{Carotenes and tocols content}

Carotene homologues (alpha and beta carotenes) were analysed using a Reverse-phase High Performance Liquid Chromatography (HPLC) (Agilent 1260 Infinity, Germany, UK) equipped with Agilent OpenLAB CDS Chemstation Edition Rev.C.01.05 (45) and analytical column JASCO CrestPak C18S, $4.6 \times 150 \mathrm{~mm}^{9)}$. Isocratic elution of mobile phase containing methanol and ethanol $(75: 25, \mathrm{v} / \mathrm{v})$ mixture was set at a flow rate of $1 \mathrm{~mL} / \mathrm{min}$. The wavelength of 292 $\mathrm{nm}$ was used to identify alpha tocopherol acetate while alpha- and beta carotenes shared similar wavelength of 450 $\mathrm{nm}$. The mean recovery of internal standard for alpha tocopherol acetate was $108.02 \pm 5.41 \%$.

Tocol homologues (i.e. tocopherols and tocotrienols) were quantified using a normal-phase High Performance Liquid Chromatography (HPLC) (Agilent 1100 series, US) fitted with fluorescence spectrophotometer, Agilent ChemStation software version B.0403-SP2 (108) and analytical column Phenomenex ${ }^{\circledR}$ Luna $5 \mu$ Silica $(250 \times 4.6 \mathrm{~mm}$ id, 5 $\mu \mathrm{M})^{9)}$. The mobile phase of tertiary mixture containing hexane, dioxane and isopropyl alcohol (Merck, Darmstadt, Germany)was used at the ratio of 970:25:5( $\mathrm{v} / \mathrm{v})$ and flow rate of $1 \mathrm{~mL} \mathrm{~min}^{-1}$. The fluorescence detector was set at excitation wavelength of $295 \mathrm{~nm}$ and emission wavelength of $325 \mathrm{~nm}$. The mean recovery of internal standard (2,2,5,7,8-pentamethyl-6-hydroxy-chromane) was $109.30 \pm$ $5.61 \%$ for red palm olein and $102.99 \pm 2.23 \%$ for palm olein. Identification of all components were done by comparing the peak areas with relation to pure standards.

\subsection{Physico-chemical analyses}

The AOCS Official Methods were employed for the determination of peroxide value (PV) $[\mathrm{Cd} 8 \mathrm{~b}-90]^{10}, p$-anisidine value $(\mathrm{AnV})[\mathrm{Cd} 18-90]^{11)}$, free fatty acid (FFA) $[\mathrm{Ca}$ 5a-40] and fatty acid composition[Ce 1a-13 $]^{12)}$. Total oxidation value (TOTOX) was calculated using the following formula: $\mathrm{TOTOX}=2 \mathrm{PV}+\mathrm{AnV}$.

\subsection{Statistical analysis}

The results were expressed as mean \pm standard deviation of duplicate heating experiment. Each quality parameter was evaluated in duplicate measurements. Therefore, analysis was performed as sextuple (triplicate measurements $\mathrm{x}$ duplicate heating experiment for physicochemical parameters) and quadruple (duplicate measurements $\mathrm{x} d u-$ 
plicate heating experiment for phytonutrients and fatty acid composition). One-way ANOVA with Tukey posthoc multiple comparison test was used to determine the difference between treatments. Pearson correlation test was used to evaluate the positive or negative interaction between parameters, type of oils and heating procedures. Statistical analysis was conducted using GraphPad Prism (version 6.00; GraphPad La Jolla, CA 9203, USA) and SPSS (version 11.5).

\section{Results and Discussion}

The temperature profile of red palm olein and palm olein during heating between 2 experiments was similar for deep-fat fryer, microwave oven, and conventional oven (Fig. 1).

Deep-fat frying transfers heat through convection directly from heating element, conventional oven also transfers heat through convection but uses air as heat transfer medium; while microwave oven transfers heat through frequency wave ${ }^{13)}$. Depletion of alpha and beta carotenes in red palm olein was found to be faster than tocols (Table 1). Similar observation was reported by Schroeder et al., and this was due to the nature of carotenes acting as the primary substrate for lipid-derived radicals or the ability of carotenes to regenerate oxidized phenols, in current case the tocols ${ }^{5)}$. Alpha and beta carotenes were found to be most stable under conventional oven heating ( $46 \%$ and $40 \%$ remaining respectively after 180 minutes of prolonged heating). Beta carotene tends to degrade faster than alpha carotene regardless of the heating conditions tested. An antioxidant synergism between carotenes and tocols could clearly be seen with microwave oven heating after total depletion of alpha and beta carotenes at $135 \mathrm{~min}$, whereby more than 3 times of steep reduction in total tocotrienols and total tocopherols were found (Table 1). Beta tocotrienol as well as beta, delta and gamma tocopherols were only detected in trace amounts in both red palm olein and palm olein, and hence no comparison was made on the thermal stability with the major tocols (alpha tocotrienol, delta tocotrienol, gamma tocotrienol and alpha tocopherol) found in both oils. Tocols were found to be very stable, evidenced by more than $70 \%$ retention after 180 hours of heating under all heating conditions. However, this was not the case for tocols in red palm olein, where close to complete depletion was observed with microwave oven heating. Tocols were found to be most heat-labile by microwave oven heating under magnetron radiation process. Malheiro et al. reported complete disappearance of alpha tocopherol in olive oil in shorter period (within 10 minutes) with microwave oven heating ${ }^{14)}$. Based on percentage depletion of tocols in red palm oil for microwave oven heating method, alpha tocotrienol was found to be the least stable followed by alpha tocopherol, gamma tocotrienol and lastly delta tocopherol. The pattern of reactivity was in accordance to the findings by Yoshida et $a l .{ }^{15}$ and alpha tocols were found to have highest absolute antioxidant activity by side chain reaction of hydrogen donation in the presence of free radicals $^{16)}$.

PV measures the oxidation or rancidity of oils during storage and heating. Peroxides are intermediate oxidation products of oil which lead to the formation of a complex mixture of volatile compounds such as aldehydes, ketones, hydrocarbons, alcohols and esters ${ }^{17}$. They have also shown to occur in oils especially at elevated temperatures as well as exposure upon to oxygen and/or light. Desired level of PV in refined palm oil is less than 0.5 meq $\mathrm{O}_{2} \mathrm{~kg}^{-1}$ and maximum level is 1 meq $\mathrm{O}_{2} \mathrm{~kg}^{-1}$, and a value higher than 10 meq $\mathrm{O}_{2} \mathrm{~kg}^{-1}$ is considered unacceptable due to rancidi$\mathrm{ty}^{18)}$. The fresh oil samples had desirable PV of 0.33 meq $\mathrm{O}_{2}$ $\mathrm{kg}^{-1}$ for red palm olein and 0.40 meq $\mathrm{O}_{2} \mathrm{~kg}^{-1}$ for palm olein (Table 2). A pattern of gradual increase in PV was observed in both test oils for all three heating techniques tested. The PV values were lowest for deep-fat fryer technique, and highest was recorded by microwave for red palm olein and conventional oven for palm olein respectively. The PVs for both samples were less than 10 meq $\mathrm{O}_{2}$ $\mathrm{kg}^{-1}$ up to 180 minutes for all three heating techniques except for microwave 11.40 meq $\mathrm{O}_{2} \mathrm{~kg}^{-1}$ which are considered as low oxidation. A PV however is less useful as hydroperoxides could decompose at $150^{\circ} \mathrm{C}^{18)}$.

The primary oxidation products are usually measured with PV test while the secondary oxidative products were quantified from AnV test. AnV represents the level of nonvolatile aldehydes, primarily, measuring presence of 2-alkenals and 2,4-alkadienals generated in the fat during the decomposition of hydroperoxides ${ }^{18)}$. Desired level of $\mathrm{AnV}$ in refined, bleached and deodorised (RBD) palm oil is $<4$ unit and maximum level is 6 unit $^{18)}$. The fresh oil samples had desirable AnV of 1.58 for red palm oil and 1.10 palm oil respectively (Table 2). In the present study AnV increased in both samples upon heat exposure.

TOTOX value gives an overview of the oxidative state of the oil by considering primary and secondary oxidation ${ }^{19)}$. TOTOX value provides a better approximation of the development of oxidative deterioration of fats and oils as it measures both hydroperoxides and secondary breakdown products. The TOTOX value of edible oils is limited to 10 unit for refined oils ${ }^{20)}$. The fresh oil samples had TOTOX value of 2.24 unit for red palm oil and 1.90 unit for palm oil respectively (Table 2). Similarly, during the heating process the TOTOX values increased significantly with heating time and the fastest deterioration was by microwave oven heating. Under similar oil heating conditions with conventional heating (by deep-fat fryer and hot plate), microwave oven and conventional oven; a similar trend of increase in hydroperoxides as secondary oxidation products are 


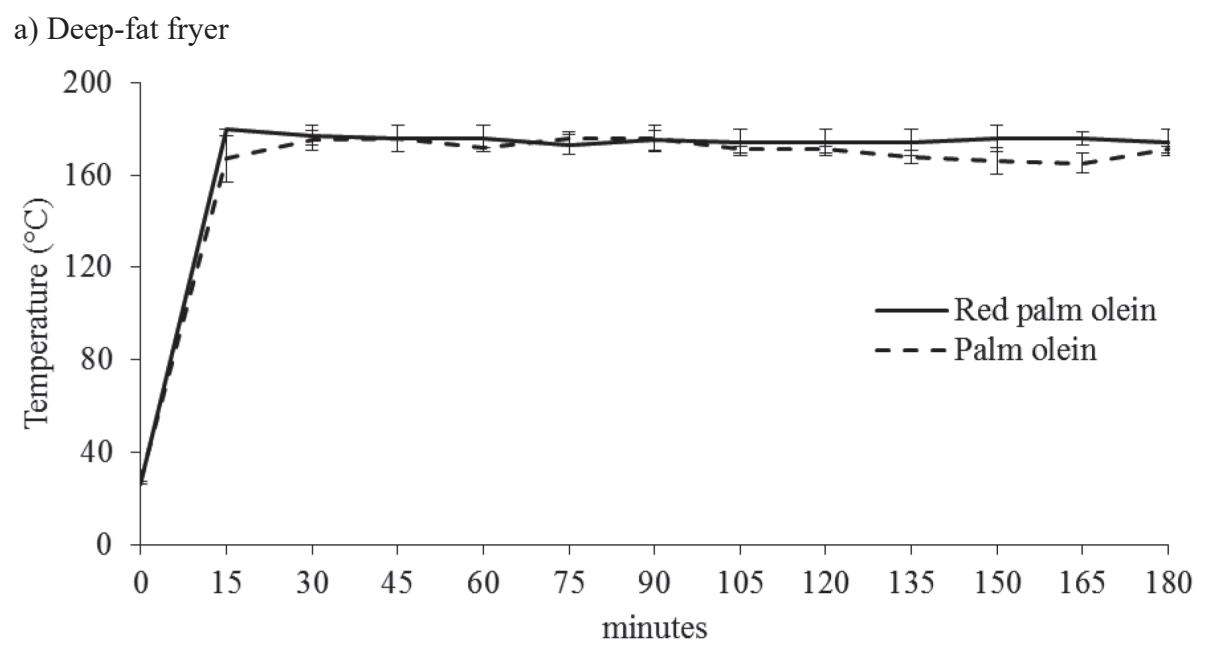

b) Microwave oven

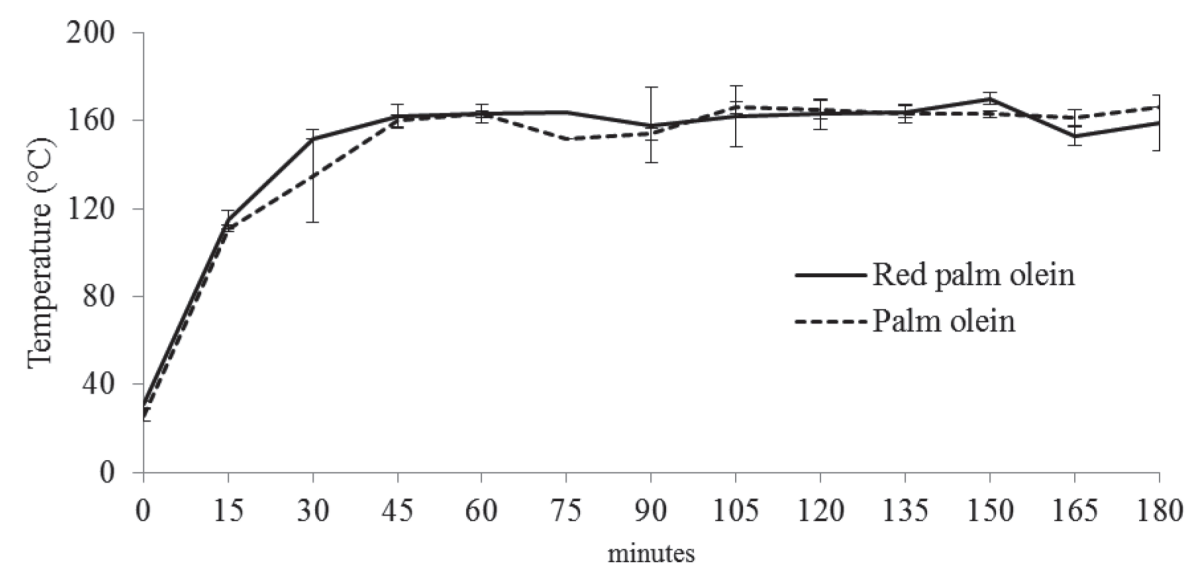

c) Conventional oven

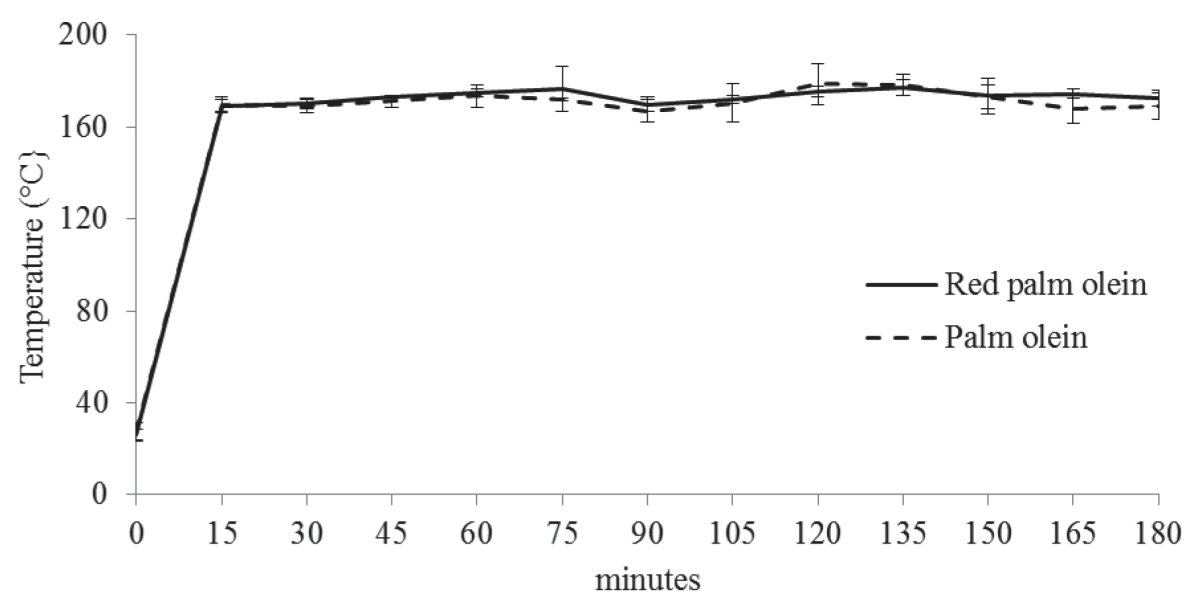

Fig. 1 Temperature profiles of red palm olein and palm olein during heating. Values are expressed as mean \pm SD for two different series of experiments. 
Table 1 Changes in phytonutrients upon heating.

a) Tocotrienol homologues and total tocotrienols

\begin{tabular}{|c|c|c|c|c|c|c|c|}
\hline \multirow{2}{*}{ Parameter } & \multirow{2}{*}{$\begin{array}{l}\text { Time } \\
(\min )\end{array}$} & \multicolumn{3}{|c|}{ i) Red palm olein } & \multicolumn{3}{|c|}{ ii) Palm olein } \\
\hline & & (a) Deep-fat fryer & (b) Microwave oven & (c) Conventional oven & (a) Deep-fat fryer & (b) Microwave oven & (c) Conventional oven \\
\hline \multirow{13}{*}{ 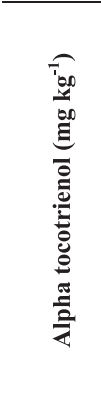 } & $\mathbf{0}$ & $268.10 \pm 4.89(\mathrm{c})$ & $268.10 \pm 4.90$ & $258.31 \pm 3.86$ (a) & $180.18 \pm 3.87$ & $180.18 \pm 3.87$ & $180.50 \pm 2.88$ \\
\hline & 15 & & 263.06 & & $20 *(\mathrm{~b})$ & $176.25 \pm 2.04(\mathrm{a}, \mathrm{c})$ & 169.0 \\
\hline & 30 & $251.25 \pm 18.55(\mathrm{~b})$ & $209.87 \pm 19.70 *(a)$ & $240.48 \pm 8.97 *$ & $163.14 \pm 0.91 *(b)$ & $157.87 \pm 0.17 *(\mathrm{a}, \mathrm{c})$ & $164.21 \pm 2.14 *(b)$ \\
\hline & 45 & $256.37 \pm 18.77(b)$ & $153.84 \pm 16.14 *(\mathrm{a}, \mathrm{c})$ & $236.08 \pm 5.29 *(b)$ & $164.46 \pm 0.67 *(b)$ & $150.70 \pm 4.36 *(a, c)$ & $166.33 \pm 1.90 *(b)$ \\
\hline & 60 & $258.98 \pm 32.90(\mathrm{~b})$ & $98.70 \pm 25.57 *(a, c)$ & $226.48 \pm 3.44 *(b)$ & $162.26 \pm 3.44 *(b)$ & $155.06 \pm 3.17 *(\mathrm{a})$ & $156.60 \pm 3.02 *$ \\
\hline & 75 & $250.87 \pm 26.55(\mathrm{~b})$ & $55.31 \pm 24.36 *(a, c)$ & $214.37 \pm 5.91 *(b)$ & $157.10 \pm 2.24 *(b, c)$ & $152.81 \pm 0.88 *(a)$ & $153.88 \pm 0.33 *(\mathrm{a})$ \\
\hline & 90 & $251.98 \pm 21.33(\mathrm{~b}, \mathrm{c})$ & $34.74 \pm 18.21 *(\mathrm{a}, \mathrm{c})$ & $214.20 \pm 5.45 *(\mathrm{a}, \mathrm{b})$ & $162.21 \pm 5.61 *(b)$ & $147.36 \pm 0.36 *(\mathrm{a})$ & $153.64 \pm 6.12 *$ \\
\hline & 105 & $267.14 \pm 22.25(\mathrm{~b}, \mathrm{c})$ & $38.19 \pm 35.78 *(a, c)$ & $212.50 \pm 1.59 *(a, b)$ & $163.87 \pm 1.92 *(b, c)$ & $147.76 \pm 1.46 *(a)$ & $151.23 \pm 1.26 *(a, b)$ \\
\hline & 120 & $264.50 \pm 4.65(\mathrm{~b}, \mathrm{c})$ & $26.74 \pm 25.31 *(a, c)$ & $205.99 \pm 4.32 *(a, b)$ & $167.66 \pm 1.75 *(b, c)$ & $141.04 \pm 5.38 *(a)$ & $147.04 \pm 4.66 *(a)$ \\
\hline & 135 & $236.66 \pm 20.64(b, c)$ & $0.00 \pm 0.00 *(a, c)$ & $201.48 \pm 3.03 *(a, b)$ & $170.27 \pm 6.93 *(b, c)$ & $142.02 \pm 3.70 *(a)$ & $145.18 \pm 2.44 *(a)$ \\
\hline & 150 & $255.29 \pm 9.81(\mathrm{~b}, \mathrm{c})$ & $3.41 \pm 3.94 *(\mathrm{a}, \mathrm{c})$ & $189.47 \pm 7.65 *(\mathrm{a}, \mathrm{b})$ & $165.82 \pm 2.83 *(\mathrm{~b}, \mathrm{c})$ & $134.08 \pm 4.99 *(\mathrm{a})$ & $145.79 \pm 0.55 *(\mathrm{a}, \mathrm{b})$ \\
\hline & 165 & $234.72 \pm 16.16(b, c)$ & $0.00 \pm 0.00 *(\mathrm{a}, \mathrm{c})$ & $181.00 \pm 14.36 *(a, b)$ & $165.05 \pm 5.69 *(b, c)$ & $134.20 \pm 2.91 *(a)$ & $140.64 \pm 1.22 *(a)$ \\
\hline & 180 & $232.48 \pm 15.21(\mathrm{~b}, \mathrm{c})$ & $2.22 \pm 2.58 *(\mathrm{a}, \mathrm{c})$ & $185.95 \pm 5.71 *(a, b)$ & $165.81 \pm 0.75 *(b, c)$ & $133.68 \pm 0.07 *(a)$ & $133.11 \pm 5.83 *(\mathrm{a})$ \\
\hline \multirow{13}{*}{ 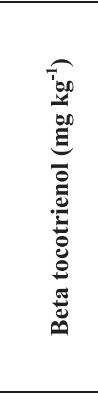 } & $\mathbf{0}$ & $22.14 \pm 0.31(\mathrm{c})$ & $22.14 \pm 0.31(\mathrm{c})$ & $21.52 \pm 0.28(\mathrm{a}, \mathrm{b})$ & $14.46 \pm 0.48(\mathrm{c})$ & $14.46 \pm 0.48(\mathrm{c})$ & $15.62 \pm 0.27(\mathrm{a}, \mathrm{b})$ \\
\hline & 15 & $21.07 \pm 1.54$ & $21.98 \pm 0.19$ & $20.81 \pm 0.28$ & $13.62 \pm 0.37(\mathrm{c})$ & $14.25 \pm 0.07$ & $14.59 \pm 0.59$ (a) \\
\hline & 30 & $21.47 \pm 1.33$ & $20.66 \pm 0.57$ & $20.52 \pm 0.64$ & $13.83 \pm 0.51$ & $13.53 \pm 0.18 *$ & $14.28 \pm 0.66$ \\
\hline & 45 & $21.03 \pm 1.08(b)$ & $18.41 \pm 0.34 *(\mathrm{a}, \mathrm{c})$ & $20.74 \pm 0.51(b)$ & $13.34 \pm 0.02 *(\mathrm{c})$ & $13.31 \pm 0.04 *(\mathrm{c})$ & $14.81 \pm 0.69(\mathrm{a}, \mathrm{b})$ \\
\hline & 60 & $21.15 \pm 1.97(b)$ & $15.98 \pm 0.83 *(a, c)$ & $20.45 \pm 0.41(\mathrm{~b})$ & $13.37 \pm 0.17 *(\mathrm{c})$ & $13.35 \pm 0.11 *(\mathrm{c})$ & $14.62 \pm 0.58(\mathrm{a}, \mathrm{b})$ \\
\hline & 75 & $20.58 \pm 1.63(\mathrm{~b})$ & $12.84 \pm 1.04 *(\mathrm{a}, \mathrm{c})$ & $19.55 \pm 0.47(b)$ & $12.95 \pm 0.12 *(\mathrm{c})$ & $13.24 \pm 0.08 *(\mathrm{c})$ & $14.26 \pm 0.73(\mathrm{a}, \mathrm{b})$ \\
\hline & 90 & $20.60 \pm 1.20(\mathrm{~b})$ & $10.86 \pm 1.60 *(\mathrm{a}, \mathrm{c})$ & $19.80 \pm 0.59(\mathrm{~b})$ & $13.27 \pm 0.20 *$ & $13.37 \pm 0.61 *$ & $14.18 \pm 0.57$ \\
\hline & 105 & $21.58 \pm 1.41(\mathrm{~b})$ & $10.20 \pm 3.91 *(\mathrm{a}, \mathrm{c})$ & $19.81 \pm 0.28(\mathrm{~b})$ & $13.38 \pm 0.30 *(\mathrm{c})$ & $13.44 \pm 0.27 *(\mathrm{c})$ & $14.53 \pm 0.76(\mathrm{a}, \mathrm{b})$ \\
\hline & 120 & o) & $9.16 \pm 3.22 *(a, c)$ & \pm 0.2 & $13.77 \pm$ & $13.03 \pm 0.53 *(\mathrm{c})$ & $14.31 \pm 0$ \\
\hline & 135 & $19.68 \pm 1.23(\mathrm{~b})$ & $4.66 \pm 0.14 *(\mathrm{a}, \mathrm{c})$ & $19.66 \pm 0.09(\mathrm{~b})$ & $13.87 \pm 0.75$ & $13.00 \pm 0.34 *$ & $13.98 \pm 0.57 *$ \\
\hline & 150 & $20.76 \pm 0.55(b, c)$ & $4.79 \pm 0.39 *(\mathrm{a}, \mathrm{c})$ & $18.96 \pm 0.68(\mathrm{a}, \mathrm{b})$ & $13.56 \pm 0.35(b)$ & $12.45 \pm 0.25 *(\mathrm{a}, \mathrm{c})$ & $14.46 \pm 0.73(\mathrm{~b})$ \\
\hline & 165 & $19.51 \pm 0.92(b)$ & $4.20 \pm 0.32 *(a, c)$ & $18.69 \pm 0.88(b)$ & $13.52 \pm 0.11 *(\mathrm{~b}, \mathrm{c})$ & $12.55 \pm 0.34 *(\mathrm{a}, \mathrm{c})$ & $14.27 \pm 0.52(\mathrm{a}, \mathrm{b})$ \\
\hline & 180 & $19.44 \pm 0.76(b)$ & $4.10 \pm 0.07 *(\mathrm{a}, \mathrm{c})$ & $18.83 \pm 0.36(b)$ & $13.36 \pm 0.24 *$ & $12.65 \pm 0.55 *$ & $13.47 \pm 0.52 *$ \\
\hline \multirow{13}{*}{ 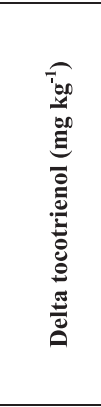 } & $\mathbf{0}$ & $115.25 \pm 0.88$ & $115.25 \pm 0.88$ & $11349+147$ & $73.12 \pm 0$ & $73.12 \pm 0.19(\mathrm{c})$ & $75.01 \pm 1.05(\mathrm{a}, \mathrm{b})$ \\
\hline & 15 & & & & & & \\
\hline & 30 & 118. & 111.4 & 109.43 & $68.06 \pm 0.49 *$ & $69.63 \pm 1$ & $69.77 \pm 3.45$ \\
\hline & 45 & $114.31 \pm 6.58(b)$ & $104.19 \pm 1.94(\mathrm{a})$ & $111.34 \pm 3.64$ & $68.12 \pm 0.03 *(c)$ & $69.02 \pm 0.65 *(\mathrm{c})$ & $72.57 \pm 1.10(\mathrm{a}, \mathrm{b})$ \\
\hline & 60 & $117.38 \pm 12.69(\mathrm{~b})$ & $95.67 \pm 1.37(\mathrm{a}, \mathrm{c})$ & 54 (b) & & $\pm 0.86 *$ & \\
\hline & 75 & $113.05 \pm 9.70(\mathrm{~b})$ & 81.92 & b) & 65.4 & $68.00=$ & $70.89 \pm 1$. \\
\hline & 90 & $113.45 \pm 6.24(b)$ & $74.75 \pm 9.92 *(a, c)$ & $108.70 \pm 3.19(\mathrm{~b})$ & $67.96 \pm 0.95 *$ & $66.35 \pm 0.32 *$ & $70.95 \pm 4.02$ \\
\hline & 105 & $.81 \pm 9.56(b)$ & $67.92 \pm 20.35 *(a, c)$ & $25 \pm 3.00(\mathrm{~b})$ & $\pm 1.24 *(\mathrm{c})$ & $66.56 \pm 0.91 *(\mathrm{c})$ & $71.89 \pm 0.65(\mathrm{a}, \mathrm{b})$ \\
\hline & 120 & $118.05 \pm 1.61(\mathrm{~b})$ & 65.15 & $106.44 \pm 0.95(\mathrm{~b})$ & $67.95 \pm 0.52 *$ & $65.03 \pm 1.94 *(\mathrm{c})$ & $70.81 \pm 3.71(b)$ \\
\hline & 135 & $108.06 \pm 6.56(b)$ & $33.87 \pm 1.02 *(\mathrm{a}, \mathrm{c})$ & $106.83 \pm 1.52(b)$ & $69.35 \pm 2.55 *(b)$ & $65.43 \pm 0.90 *(\mathrm{a}, \mathrm{c})$ & $70.67 \pm 2.35(b)$ \\
\hline & 150 & $116.83 \pm 1.86(b, c)$ & $33.75 \pm 7.70 *(\mathrm{a}, \mathrm{c})$ & $104.61 \pm 3.41 *(\mathrm{a}, \mathrm{b})$ & $*(\mathrm{~b})$ & $*(a, c)$ & $70.14 \pm 0.99(b)$ \\
\hline & 165 & $107.57 \pm 5.38(\mathrm{~b})$ & $24.51 \pm 1.77 *(a, c)$ & $104.83 \pm 4.60 *(b)$ & $67.48 \pm 1.60 *(b)$ & $62.58 \pm 0.88 *(a, c)$ & $70.31 \pm 3.03(\mathrm{~b})$ \\
\hline & 180 & $107.33 \pm 5.47(b)$ & $19.31 \pm 1.70 *(\mathrm{a}, \mathrm{c})$ & $104.21 \pm 1.34 *(b)$ & $66.27 \pm 0.97 *$ & $62.94 \pm 0.83 *(c)$ & $67.69 \pm 2.85 *(b)$ \\
\hline \multirow{13}{*}{ 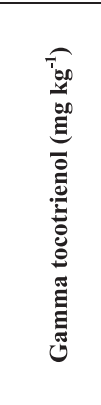 } & $\mathbf{0}$ & $336.45 \pm$ & $336.44 \pm 6.45(\mathrm{c})$ & $=4$ & 219. & $219.50 \pm 5.45$ & 22 \\
\hline & 15 & $.07 \pm 27.92$ & $332.49 \pm 3.49$ & $.56 \pm 4.73$ & $200.79 \pm 3.54 *(b, c)$ & $217.05 \pm 0$ & $206.63 \pm 1.52 *(a, b)$ \\
\hline & 30 & $316.00 \pm 25.14$ & $301.37 \pm 12.06$ & $307.85 \pm 10.66$ & $196.32 \pm 1.05 *$ & $200.07 \pm$ & $203.15 \pm$ \\
\hline & 45 & $303.86 \pm 19.66(b)$ & $239.52 \pm 2.89 *(a, c)$ & $308.79 \pm 8.12(b)$ & $196.24 \pm 0.46 *(b, c)$ & $190.00 \pm 2.10 *(a, c)$ & $209.70 \pm 2.25 *(a, b)$ \\
\hline & 60 & $303.27 \pm 35.97(b)$ & $169.14 \pm 19.96 *(a, c)$ & $301.57 \pm 5.63 *(b)$ & $192.24 \pm 4.15 *(\mathrm{c})$ & $187.39 \pm 2.78 *(\mathrm{c})$ & $200.99 \pm 4.41 *(a, b)$ \\
\hline & 75 & \pm 29.04 (b) & $*(\mathrm{a}, \mathrm{c})$ & *(b) & $*(\mathrm{c})$ & 182.21 & $200.73 \pm 1.41 *(a, b)$ \\
\hline & 90 & $288.23 \pm 22.68(\mathrm{~b})$ & $62.10 \pm 27.23 *(a, c)$ & $286.71 \pm 11.42 *(b)$ & $191.54 \pm 5.93 *(b)$ & $173.74 \pm 1.89 *(\mathrm{a}, \mathrm{c})$ & $200.62 \pm 6.80 *(b)$ \\
\hline & 105 & $299.60 \pm 24.57(\mathrm{~b})$ & $64.30 \pm 57.23 *(a, c)$ & $286.72 \pm 2.59 *(b)$ & $192.80 \pm 1.84 *(b, c)$ & $170.75 \pm 0.96 *(a, c)$ & $201.27 \pm 1.91 *(a, b)$ \\
\hline & 120 & $294.84 \pm 2.87(\mathrm{~b})$ & $1.50 *(\mathrm{a}, \mathrm{c})$ & $4.96 *(\mathrm{~b})$ & *(b) & $160.35 \pm 6$ & $196.74 \pm 4.62 *(\mathrm{~b})$ \\
\hline & 135 & $260.63 \pm 21.36 *(b)$ & $5.19 \pm 0.34 *(\mathrm{a}, \mathrm{c})$ & $280.63 \pm 3.39 *(b)$ & $197.96 \pm 8.16 *(b)$ & $159.25 \pm 2.53 *(\mathrm{a}, \mathrm{c})$ & $194.72 \pm 2.11 *(b)$ \\
\hline & 150 & $275.42 \pm 12.00 *(b)$ & $5.54 \pm 1.69 *(\mathrm{a}, \mathrm{c})$ & $264.34 \pm 14.91 *(b)$ & $192.38 \pm 3.60 *(b)$ & $148.59 \pm 2.00 *(a, c)$ & $196.65 \pm 0.43 *(b)$ \\
\hline & 165 & $250.90 \pm 16.53 *(b)$ & $4.15 \pm 0.06 *(\mathrm{a}, \mathrm{c})$ & $253.48 \pm 15.14 *(b)$ & $191.19 \pm 5.93 *(b)$ & $144.94 \pm 0.70 *(\mathrm{a}, \mathrm{c})$ & $193.97 \pm 4.62 *(b)$ \\
\hline & 180 & $244.98 \pm 14.75 *(b)$ & $2.11 \pm 2.43 *(\mathrm{a}, \mathrm{c})$ & $257.98 \pm 10.36 *(b)$ & $189.37 \pm 1.91 *(b, c)$ & $142.90 \pm 1.38 *(a, c)$ & $181.18 \pm 4.34 *(\mathrm{a}, \mathrm{b})$ \\
\hline \multirow{13}{*}{ 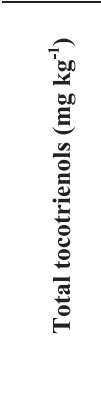 } & $\mathbf{0}$ & $74193+1247(\mathrm{c})$ & $741.93 \pm 12.47(\mathrm{c})$ & $71769+845(\mathrm{ab})$ & $48725+975$ & $487.25 \pm 9.75$ & $49262+416$ \\
\hline & 15 & $698.37 \pm 64.14$ & $732.6 \pm 5.34$ & $695.15 \pm 12.27$ & $449.23 \pm 7.96 *(b, c)$ & $480.91 \pm 2.83(\mathrm{a}, \mathrm{c})$ & $461.05 \pm 2.87 *(a, b)$ \\
\hline & 30 & $706.89 \pm 47.19$ & $643.32 \pm 34.08$ & $67828+2423$ & $441.35 \pm 2.6 *$ & $441.11 \pm 4.35 *$ & $451.42 \pm 11.11 *$ \\
\hline & 45 & $695.58 \pm 46.08(\mathrm{~b})$ & $9 *(a, c)$ & $676.94 \pm 1$ & $442.16 \pm 1.18 *(b, c)$ & $423.03 \pm 6.29 *(\mathrm{a}, \mathrm{c})$ & $463.42 \pm 3.93 *(a, b)$ \\
\hline & 60 & $700.78 \pm 83.49$ (b) & $379.49 \pm 47.73 *(\mathrm{a}, \mathrm{c})$ & $659.01 \pm 9.53(\mathrm{~b})$ & $434.03 \pm 9.62 *$ & $424.59 \pm 6.75 *(\mathrm{c})$ & $442.24 \pm 7.71 *(\mathrm{~b})$ \\
\hline & 75 & $673.74 \pm 66.87(b)$ & $250.44 \pm 44.86 *(\mathrm{a}, \mathrm{c})$ & $625.07 \pm 17.37(b)$ & $420.59 \pm 4.32 *(\mathrm{c})$ & $416.26 \pm 4.4 *(\mathrm{c})$ & $439.76 \pm 3.01 *(a, b)$ \\
\hline & 90 & $674.26 \pm 51.35(\mathrm{~b})$ & $182.44 \pm 56.5 *(\mathrm{a}, \mathrm{c})$ & $629.41 \pm 20.62(b)$ & $434.98 \pm 12.63 *(b)$ & $400.82 \pm 2.99 *(\mathrm{a}, \mathrm{c})$ & $439.39 \pm 16.92 *(b)$ \\
\hline & 105 & $707.12 \pm 57.76(\mathrm{~b})$ & $180.62 \pm 117.26 *(\mathrm{a}, \mathrm{c})$ & $626.28 \pm 5.99(\mathrm{~b})$ & $437.65 \pm 4.94 *(\mathrm{~b})$ & $398.51 \pm 2.79 *(\mathrm{a}, \mathrm{c})$ & $438.91 \pm 2.99 *(\mathrm{~b})$ \\
\hline & 120 & $698.82 \pm 8.87(\mathrm{~b})$ & $148.65 \pm 87.61 *(\mathrm{a}, \mathrm{c})$ & $616.49 \pm 10.19(b)$ & $444.8 \pm 4.36 *(b)$ & $379.45 \pm 14.65 *(a, c)$ & $428.9 \pm 12.47 *(b)$ \\
\hline & 135 & $625.04 \pm 49.72(b)$ & $43.72 \pm 1.18 *(\mathrm{a}, \mathrm{c})$ & $608.61 \pm 5.04(\mathrm{~b})$ & $451.45 \pm 18.31 *(b, c)$ & $379.7 \pm 6.97 *(a, c)$ & $424.55 \pm 6.53 *(a, b)$ \\
\hline & 150 & $668.3 \pm 24.17(\mathrm{~b})$ & $47.49 \pm 13.69 *(\mathrm{a}, \mathrm{c})$ & $641.63 \pm 145.05(\mathrm{~b})$ & $440.12 \pm 6.75 *(b, c)$ & $358.58 \pm 6.63 *(a, c)$ & $427.04 \pm 1 *(a, b)$ \\
\hline & 165 & $612.69 \pm 38.9 *(b)$ & $32.86 \pm 2.08 *(\mathrm{a}, \mathrm{c})$ & $558 \pm 34.93 *(\mathrm{~b})$ & $437.24 \pm 11.95 *(b, c)$ & $354.26 \pm 3.97 *(a, c)$ & $419.19 \pm 8.36 *(a, b)$ \\
\hline & 180 & $604.23 \pm 36.18 *(\mathrm{~b})$ & $27.73 \pm 6.63 *(a, c)$ & $566.97 \pm 17.6 *(b)$ & $434.8 \pm 3.48 *(b, c)$ & $352.18 \pm 2.64 *(a, c)$ & $395.45 \pm 12.68 *(a, b)$ \\
\hline
\end{tabular}


Table 1 Continued.

b) Tocopherol homologues and total tocopherols

\begin{tabular}{|c|c|c|c|c|c|c|c|}
\hline \multirow{2}{*}{ Parameter } & \multirow{2}{*}{$\begin{array}{l}\text { Time } \\
\text { (min) }\end{array}$} & \multicolumn{3}{|c|}{ i) Red palm olein } & \multicolumn{3}{|c|}{ ii) Palm olein } \\
\hline & & (a) Deep-fat fryer & (b) Microwave oven & (c) Conventional oven & (a) Deep-fat fryer & (b) Microwave oven & (c) Conventional oven \\
\hline \multirow{13}{*}{ 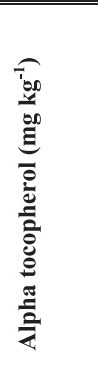 } & 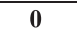 & $161.64 \pm 3.72$ & $162.14 \pm 3.20$ & $157.48 \pm 1.70$ & $126.46 \pm 1.32$ & $126.82 \pm 2.80$ & $126.34 \pm 1.53$ \\
\hline & 15 & $164.27 \pm 6.42(\mathrm{c})$ & $158.46 \pm 1.10$ & $151.62 \pm 1.77(\mathrm{a})$ & $116.75 \pm 2.20 *(b)$ & $123.15 \pm 0.83(a, c)$ & $118.40 \pm 0.19 *(b)$ \\
\hline & 30 & $161.41 \pm 13.90(\mathrm{~b})$ & $129.34 \pm 10.72 *(a)$ & $148.04 \pm 5.06^{*}$ & $114.84 \pm 0.40 *$ & $112.51 \pm 0.40 *$ & $115.57 \pm 2.85 *$ \\
\hline & 45 & $158.64 \pm 11.29(\mathrm{~b})$ & $98.58 \pm 10.44 *(a, c)$ & $147.65 \pm 4.76 *(b)$ & $117.00 \pm 0.16 *(b)$ & $107.00 \pm 2.30 *(a, c)$ & $117.09 \pm 1.02 *(b)$ \\
\hline & 60 & $161.02 \pm 19.74(\mathrm{~b})$ & $65.53 \pm 16.09 *(a, c)$ & $142.98 \pm 1.67 *(b)$ & $116.51 \pm 1.15 *(\mathrm{c})$ & $113.29 \pm 2.11 *$ & $110.61 \pm 2.15 *(a)$ \\
\hline & 75 & $155.80 \pm 16.21(\mathrm{~b})$ & $38.34 \pm 16.27 *(\mathrm{a}, \mathrm{c})$ & $133.64 \pm 2.88 *(b)$ & $112.64 \pm 1.54 *(\mathrm{c})$ & $111.56 \pm 0.61 *(\mathrm{c})$ & $108.70 \pm 0.15 *(a, b)$ \\
\hline & 90 & $157.58 \pm 12.92(\mathrm{~b}, \mathrm{c})$ & $24.72 \pm 11.61 *(\mathrm{a}, \mathrm{c})$ & $134.83 \pm 4.03 *(a, b)$ & $117.69 \pm 4.56 *(b, c)$ & $108.78 \pm 0.24 *(a)$ & $109.33 \pm 4.25 *(a)$ \\
\hline & 105 & $167.10 \pm 13.78(b, c)$ & $26.43 \pm 24.12 *(a, c)$ & $132.25 \pm 1.07 *(a, b)$ & $116.61 \pm 0.72 *(b, c)$ & $110.81 \pm 1.09 *(\mathrm{a}, \mathrm{c})$ & $108.41 \pm 0.72 *(a, b)$ \\
\hline & 120 & $167.12 \pm 1.90(b, c)$ & $19.12 \pm 16.98 *(\mathrm{a}, \mathrm{c})$ & $127.15 \pm 2.37 *(a, b)$ & $120.20 \pm 0.93 *(b, c)$ & $108.36 \pm 3.28 *(a)$ & $105.49 \pm 2.97 *(\mathrm{a})$ \\
\hline & 135 & $150.99 \pm 11.99(b, c)$ & $0.00 \pm 0.00 *(\mathrm{a}, \mathrm{c})$ & $125.62 \pm 2.03 *(a, b)$ & $122.50 \pm 4.73(b, c)$ & $109.16 \pm 2.29 *(a)$ & $105.11 \pm 2.25 *(\mathrm{a})$ \\
\hline & 150 & $162.53 \pm 6.73(b, c)$ & $0.00 \pm 0.00 *(\mathrm{a}, \mathrm{c})$ & $121.50 \pm 3.01 *(a, b)$ & $120.87 \pm 1.68(\mathrm{~b}, \mathrm{c})$ & $104.26 \pm 3.50 *(\mathrm{a})$ & $105.45 \pm 0.39 *(a)$ \\
\hline & 165 & $151.09 \pm 9.78(\mathrm{~b}, \mathrm{c})$ & $0.00 \pm 0.00 *(\mathrm{a}, \mathrm{c})$ & $123.51 \pm 0.22 *(\mathrm{a}, \mathrm{b})$ & $119.43 \pm 4.19 *(b, c)$ & $104.94 \pm 2.08 *(\mathrm{a})$ & $102.21 \pm 1.24 *(a)$ \\
\hline & 180 & $151.21 \pm 8.41(\mathrm{~b}, \mathrm{c})$ & $0.00 \pm 0.00 *(\mathrm{a}, \mathrm{c})$ & $116.07 \pm 3.06 *(\mathrm{a}, \mathrm{b})$ & $120.18 \pm 0.72 *(b, c)$ & $104.85 \pm 0.32 *(\mathrm{a}, \mathrm{c})$ & $97.20 \pm 3.75 *(a, b)$ \\
\hline \multirow{13}{*}{ 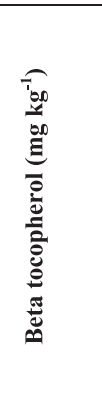 } & 0 & $5.57 \pm 0.05(\mathrm{c})$ & $5.57 \pm 0.05(\mathrm{c})$ & $5.33 \pm 0.17(\mathrm{a}, \mathrm{b})$ & $4.91 \pm 0.06(\mathrm{c})$ & $4.91 \pm 0.06(\mathrm{c})$ & $4.72 \pm 0.04(\mathrm{a}, \mathrm{b})$ \\
\hline & 15 & $6.20 \pm 0.22 *(b, c)$ & $5.60 \pm 0.05(\mathrm{a})$ & $5.37 \pm 0.18(\mathrm{a})$ & $4.93 \pm 0.10(\mathrm{c})$ & $4.90 \pm 0.10$ & $4.75 \pm 0.07(\mathrm{a})$ \\
\hline & 30 & $6.61 \pm 0.08 *(b, c)$ & $5.63 \pm 0.04(\mathrm{a}, \mathrm{c})$ & $5.41 \pm 0.07(\mathrm{a}, \mathrm{b})$ & $5.02 \pm 0.17(\mathrm{c})$ & $4.99 \pm 0.06(\mathrm{c})$ & $4.76 \pm 0.05(\mathrm{a}, \mathrm{b})$ \\
\hline & 45 & $6.73 \pm 0.13 *(b, c)$ & $5.70 \pm 0.12(\mathrm{a}, \mathrm{c})$ & $5.38 \pm 0.06(\mathrm{a}, \mathrm{b})$ & $4.83 \pm 0.08(b)$ & $4.99 \pm 0.06(\mathrm{a}, \mathrm{c})$ & $4.78 \pm 0.06(b)$ \\
\hline & 60 & $6.97 \pm 0.23 *(b, c)$ & $5.75 \pm 0.12(\mathrm{a})$ & $5.51 \pm 0.01(\mathrm{a}, \mathrm{b})$ & $4.94 \pm 0.24$ & $5.07 \pm 0.09(\mathrm{c})$ & $4.76 \pm 0.08(b)$ \\
\hline & 75 & $6.90 \pm 0.15 *(b, c)$ & $5.76 \pm 0.28(\mathrm{a}, \mathrm{c})$ & $5.43 \pm 0.09(\mathrm{a}, \mathrm{b})$ & $4.87 \pm 0.23$ & $5.09 \pm 0.06(\mathrm{c})$ & $4.80 \pm 0.09(b)$ \\
\hline & 90 & $6.93 \pm 0.10 *(b, c)$ & $5.62 \pm 0.37(\mathrm{a}, \mathrm{c})$ & $5.64 \pm 0.16 *(a)$ & $4.95 \pm 0.17$ & $5.16 \pm 0.14(\mathrm{c})$ & $4.73 \pm 0.15(b)$ \\
\hline & 105 & $7.01 \pm 0.10 *(b, c)$ & $5.95 \pm 0.11(\mathrm{a}, \mathrm{c})$ & $5.66 \pm 0.05 *(a, b)$ & $4.96 \pm 0.18$ & $5.09 \pm 0.05(\mathrm{c})$ & $4.87 \pm 0.03(b)$ \\
\hline & 120 & $7.02 \pm 0.16 *(b, c)$ & $5.72 \pm 0.44(a)$ & $5.60 \pm 0.11(\mathrm{a})$ & $5.06 \pm 0.13(\mathrm{c})$ & $5.14 \pm 0.06(\mathrm{c})$ & $4.76 \pm 0.11(\mathrm{a}, \mathrm{b})$ \\
\hline & 135 & $6.82 \pm 0.04 *(b, c)$ & $5.09 \pm 0.22(\mathrm{a}, \mathrm{c})$ & $5.56 \pm 0.08(\mathrm{a}, \mathrm{b})$ & $5.04 \pm 0.15(\mathrm{c})$ & $5.17 \pm 0.06 *(\mathrm{c})$ & $4.84 \pm 0.04(\mathrm{a}, \mathrm{b})$ \\
\hline & 150 & $6.90 \pm 0.15 *(b, c)$ & $5.12 \pm 0.33(\mathrm{a}, \mathrm{c})$ & $5.64 \pm 0.20 *(a, b)$ & $5.17 \pm 0.03(\mathrm{c})$ & $5.16 \pm 0.06 *(\mathrm{c})$ & $4.79 \pm 0.06(\mathrm{a}, \mathrm{b})$ \\
\hline & 165 & $6.75 \pm 0.03 *(b)$ & $2.58 \pm 2.98 *(\mathrm{a}, \mathrm{c})$ & $5.82 \pm 0.09 *(b)$ & $5.16 \pm 0.02$ & $5.01 \pm 0.15$ & $4.89 \pm 0.19$ \\
\hline & 180 & $6.66 \pm 0.06 *(b)$ & $2.43 \pm 2.81 *(\mathrm{a}, \mathrm{c})$ & $5.75 \pm 0.07 *(b)$ & $5.11 \pm 0.02(\mathrm{c})$ & $5.13 \pm 0.22(\mathrm{c})$ & $4.75 \pm 0.13(\mathrm{a}, \mathrm{b})$ \\
\hline \multirow{13}{*}{ 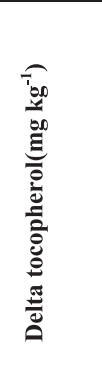 } & 0 & $17.90 \pm 0.15$ & $17.90 \pm 0.15$ & $17.67 \pm 0.32$ & $4.05 \pm 0.08$ & $4.05 \pm 0.08$ & $4.06 \pm 0.07$ \\
\hline & 15 & $17.84 \pm 1.19$ & $17.92 \pm 0.20$ & $17.63 \pm 0.33$ & $4.03 \pm 0.04$ & $4.01 \pm 0.04$ & $3.96 \pm 0.04$ \\
\hline & 30 & $18.46 \pm 1.25$ & $17.61 \pm 0.10$ & $17.38 \pm 0.53$ & $3.97 \pm 0.09$ & $3.97 \pm 0.06$ & $3.96 \pm 0.03$ \\
\hline & 45 & $18.07 \pm 0.96$ & $16.84 \pm 0.16$ & $17.67 \pm 0.53$ & $3.98 \pm 0.10$ & $4.00 \pm 0.04$ & $4.04 \pm 0.04$ \\
\hline & 60 & $18.41 \pm 1.65(\mathrm{~b})$ & $16.11 \pm 0.42(a)$ & $17.68 \pm 0.31$ & $3.98 \pm 0.07$ & $3.98 \pm 0.08$ & $3.96 \pm 0.05$ \\
\hline & 75 & $17.97 \pm 1.35(\mathrm{~b})$ & $14.23 \pm 0.32 *(\mathrm{a}, \mathrm{c})$ & $16.91 \pm 0.44(b)$ & $3.95 \pm 0.06$ & $3.93 \pm 0.04$ & $4.00 \pm 0.08$ \\
\hline & 90 & $17.94 \pm 1.06(b)$ & $13.47 \pm 1.04 *(\mathrm{a}, \mathrm{c})$ & $17.18 \pm 0.49(b)$ & $3.97 \pm 0.08$ & $3.96 \pm 0.04$ & $4.05 \pm 0.18$ \\
\hline & 105 & $18.67 \pm 1.22(\mathrm{~b})$ & $12.52 \pm 2.38 *(\mathrm{a}, \mathrm{c})$ & $17.13 \pm 0.40(b)$ & $3.91 \pm 0.02(\mathrm{c})$ & $4.00 \pm 0.01$ & $4.07 \pm 0.09(\mathrm{a})$ \\
\hline & 120 & $18.73 \pm 0.09(\mathrm{~b})$ & $12.15 \pm 2.19 *(\mathrm{a}, \mathrm{c})$ & $17.03 \pm 0.06(b)$ & $3.96 \pm 0.03$ & $3.93 \pm 0.02$ & $4.06 \pm 0.15$ \\
\hline & 135 & $17.40 \pm 0.84(b)$ & $8.27 \pm 0.23 *(a, c)$ & $17.08 \pm 0.23(b)$ & $4.00 \pm 0.11$ & $3.93 \pm 0.07$ & $4.04 \pm 0.13$ \\
\hline & 150 & $18.64 \pm 0.34(\mathrm{~b}, \mathrm{c})$ & $8.17 \pm 0.99 *(\mathrm{a}, \mathrm{c})$ & $16.90 \pm 0.47(\mathrm{a}, \mathrm{b})$ & $3.97 \pm 0.07$ & $3.94 \pm 0.05$ & $4.05 \pm 0.11$ \\
\hline & 165 & $17.43 \pm 0.92(b)$ & $7.01 \pm 0.22 *(\mathrm{a}, \mathrm{c})$ & $16.94 \pm 0.86(\mathrm{~b})$ & $3.95 \pm 0.02$ & $3.93 \pm 0.04$ & $4.05 \pm 0.16$ \\
\hline & 180 & $17.39 \pm 0.89(\mathrm{~b})$ & $6.25 \pm 0.30 *(a, c)$ & $16.82 \pm 0.10(b)$ & $3.99 \pm 0.06$ & $3.97 \pm 0.07$ & $3.99 \pm 0.10$ \\
\hline \multirow{13}{*}{ 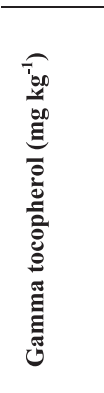 } & 0 & $42.63 \pm 0.56(\mathrm{c})$ & $42.62 \pm 0.57(\mathrm{c})$ & $40.83 \pm 0.42(\mathrm{a}, \mathrm{b})$ & $6.85 \pm 0.23(\mathrm{c})$ & $6.84 \pm 0.23(\mathrm{c})$ & $7.65 \pm 0.10(a, b)$ \\
\hline & 15 & $39.33 \pm 3.55$ & $42.35 \pm 0.42$ & $39.70 \pm 0.64$ & $6.51 \pm 0.15(\mathrm{c})$ & $6.83 \pm 0.11(\mathrm{c})$ & $7.30 \pm 0.23(\mathrm{a}, \mathrm{b})$ \\
\hline & 30 & $40.17 \pm 3.12$ & $39.03 \pm 1.36$ & $39.01 \pm 1.33$ & $6.66 \pm 0.32$ & $6.59 \pm 0.15$ & $7.15 \pm 0.35$ \\
\hline & 45 & $38.75 \pm 2.42(b)$ & $31.97 \pm 0.40 *(\mathrm{a}, \mathrm{c})$ & $39.51 \pm 1.32(\mathrm{~b})$ & $6.31 \pm 0.01 *(\mathrm{c})$ & $6.39 \pm 0.04(\mathrm{c})$ & $7.38 \pm 0.45(\mathrm{a}, \mathrm{b})$ \\
\hline & 60 & $38.87 \pm 4.26(\mathrm{~b})$ & $23.87 \pm 2.48 *(\mathrm{a}, \mathrm{c})$ & $38.85 \pm 1.03(\mathrm{~b})$ & $6.38 \pm 0.08(\mathrm{c})$ & $6.44 \pm 0.07(c)$ & $7.38 \pm 0.40(a, b)$ \\
\hline & 75 & $37.35 \pm 3.39(\mathrm{~b})$ & $15.70 \pm 2.31 *(\mathrm{a}, \mathrm{c})$ & $36.63 \pm 1.06 *(\mathrm{~b})$ & $6.25 \pm 0.15 *(\mathrm{c})$ & $6.34 \pm 0.06 *(\mathrm{c})$ & $7.16 \pm 0.44(a, b)$ \\
\hline & 90 & $37.11 \pm 2.64(b)$ & $11.01 \pm 3.50 *(\mathrm{a}, \mathrm{c})$ & $37.07 \pm 1.34 *(b)$ & $6.36 \pm 0.07$ & $6.48 \pm 0.32$ & $6.91 \pm 0.40$ \\
\hline & 105 & $38.40 \pm 2.89(b)$ & $11.28 \pm 7.20 *(\mathrm{a}, \mathrm{c})$ & $36.84 \pm 0.30 *(b)$ & $6.33 \pm 0.21 *(\mathrm{c})$ & $6.46 \pm 0.12$ & $7.15 \pm 0.61(\mathrm{a})$ \\
\hline & 120 & $38.07 \pm 0.28(b)$ & $9.23 \pm 5.30 *(\mathrm{a}, \mathrm{c})$ & $36.63 \pm 0.77 *(b)$ & $6.50 \pm 0.27$ & $6.22 \pm 0.26 *$ & $6.95 \pm 0.56$ \\
\hline & 135 & $34.20 \pm 2.54 *(b)$ & $2.02 \pm 2.33 *(\mathrm{a}, \mathrm{c})$ & $36.32 \pm 0.56 *(b)$ & $6.49 \pm 0.34$ & $6.16 \pm 0.16 *$ & $6.83 \pm 0.51$ \\
\hline & 150 & $35.94 \pm 1.23(\mathrm{~b})$ & $2.06 \pm 2.38 *(\mathrm{a}, \mathrm{c})$ & $34.48 \pm 1.55 *(b)$ & $6.52 \pm 0.22$ & $5.96 \pm 0.15 *(\mathrm{c})$ & $6.95 \pm 0.63(\mathrm{~b})$ \\
\hline & 165 & $33.07 \pm 1.92 *(b)$ & $3.05 \pm 2.08 *(\mathrm{a}, \mathrm{c})$ & $33.46 \pm 1.74 *(b)$ & $6.45 \pm 0.13$ & $5.97 \pm 0.19 *(\mathrm{c})$ & $6.95 \pm 0.58(b)$ \\
\hline & 180 & $32.50 \pm 1.56 *(b)$ & $0.94 \pm 1.87 *(\mathrm{a}, \mathrm{c})$ & $33.78 \pm 1.36 *(b)$ & $6.30 \pm 0.15 *$ & $6.03 \pm 0.29 *$ & $6.58 \pm 0.50$ \\
\hline \multirow{13}{*}{ 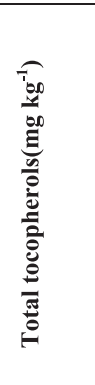 } & 0 & $227.74 \pm 3.24(\mathrm{c})$ & $228.24 \pm 3.88(\mathrm{c})$ & $221.31 \pm 2.2(\mathrm{~b}, \mathrm{a})$ & $142.27 \pm 1.48$ & $142.62 \pm 2.94$ & $142.77 \pm 1.6$ \\
\hline & 15 & $227.63 \pm 11.3$ & $224.32 \pm 1.76$ & $214.33 \pm 2.37$ & $132.22 \pm 2.37 *(b)$ & $138.9 \pm 0.76(\mathrm{a}, \mathrm{c})$ & $134.41 \pm 0.19 *(b)$ \\
\hline & 30 & $226.65 \pm 18.34(b)$ & $191.62 \pm 12.19(\mathrm{a})$ & $209.84 \pm 6.51 *$ & $130.5 \pm 0.36 *$ & $128.06 \pm 0.6 *(\mathrm{c})$ & $131.45 \pm 2.74 *(b)$ \\
\hline & 45 & $222.2 \pm 14.79(\mathrm{~b})$ & $153.09 \pm 10.74 *(\mathrm{a}, \mathrm{c})$ & $210.21 \pm 6.2 *(b)$ & $132.13 \pm 0.35 *(b)$ & $122.39 \pm 2.33 *(\mathrm{a}, \mathrm{c})$ & $133.29 \pm 1.47 *(\mathrm{a})$ \\
\hline & 60 & $225.28 \pm 25.87(b)$ & $111.26 \pm 19.01 *(\mathrm{a}, \mathrm{c})$ & $205.02 \pm 2.54 *(\mathrm{~b})$ & $131.82 \pm 1.03 *(\mathrm{c})$ & $128.77 \pm 2.17 *$ & $126.69 \pm 2.58 *(\mathrm{a})$ \\
\hline & 75 & $218.02 \pm 21.1(\mathrm{~b})$ & $74.03 \pm 19.16 *(a, c)$ & $192.6 \pm 3.77 *(b)$ & $127.71 \pm 1.4 *(\mathrm{c})$ & $126.93 \pm 0.64 *(\mathrm{c})$ & $124.66 \pm 0.45 *(\mathrm{~b}, \mathrm{a})$ \\
\hline & 90 & $219.56 \pm 16.66(\mathrm{~b}, \mathrm{c})$ & $54.82 \pm 16.06 *(\mathrm{a}, \mathrm{c})$ & $194.73 \pm 5.39 *(a, b)$ & $132.96 \pm 4.51 *(\mathrm{~b}, \mathrm{c})$ & $124.38 \pm 0.62 *(\mathrm{a})$ & $125.02 \pm 4.02 *(a)$ \\
\hline & 105 & $231.19 \pm 17.99(\mathrm{~b})$ & $56.18 \pm 33.81 *(\mathrm{a}, \mathrm{c})$ & $191.88 \pm 1.58 *(b)$ & $131.81 \pm 1.03 *(b, c)$ & $126.36 \pm 1.05 *(\mathrm{a}, \mathrm{c})$ & $124.49 \pm 0.74 *(a, b)$ \\
\hline & 120 & $230.93 \pm 2.22(\mathrm{~b}, \mathrm{c})$ & $46.23 \pm 24.85 *(\mathrm{a}, \mathrm{c})$ & $186.41 \pm 2.97 *(a, b)$ & $135.72 \pm 1.24 *(\mathrm{~b}, \mathrm{c})$ & $123.65 \pm 3.49 *(a)$ & $121.27 \pm 2.46 *(a)$ \\
\hline & 135 & $209.42 \pm 15.4(\mathrm{~b}, \mathrm{c})$ & $15.38 \pm 2.46 *(\mathrm{a}, \mathrm{c})$ & $184.57 \pm 2.5 *(a, b)$ & $138.03 \pm 5.29(\mathrm{~b}, \mathrm{c})$ & $124.42 \pm 2.39 *(\mathrm{a})$ & $120.82 \pm 2.04 *(\mathrm{a})$ \\
\hline & 150 & $224.02 \pm 8.14(\mathrm{~b}, \mathrm{c})$ & $15.34 \pm 3.52 *(\mathrm{a}, \mathrm{c})$ & $178.51 \pm 4.16 *(\mathrm{a}, \mathrm{b})$ & $136.53 \pm 1.38(\mathrm{~b}, \mathrm{c})$ & $119.32 \pm 3.56 *(\mathrm{a})$ & $121.24 \pm 0.48 *(\mathrm{a})$ \\
\hline & 165 & $208.34 \pm 12.59(\mathrm{~b}, \mathrm{c})$ & $12.64 \pm 4.25 *(\mathrm{a}, \mathrm{c})$ & $179.72 \pm 2.77 *(a, b)$ & $134.99 \pm 4.07 *(b, c)$ & $119.86 \pm 2.13 *(\mathrm{a})$ & $118.11 \pm 0.92 *(\mathrm{a})$ \\
\hline & 180 & $207.75 \pm 10.81(\mathrm{~b}, \mathrm{c})$ & $9.61 \pm 4.51 *(\mathrm{a}, \mathrm{c})$ & $172.42 \pm 4.08 *(\mathrm{a}, \mathrm{b})$ & $135.59 \pm 0.75 *(\mathrm{~b}, \mathrm{c})$ & $119.97 \pm 0.46 *(\mathrm{a}, \mathrm{c})$ & $112.52 \pm 3.29 *(a, b)$ \\
\hline
\end{tabular}


Table 1 Continued.

c) Total tocols, alpha- and beta-carotenes

\begin{tabular}{|c|c|c|c|c|c|c|c|}
\hline \multirow{2}{*}{ Parameter } & \multirow{2}{*}{$\begin{array}{l}\text { Time } \\
(\min )\end{array}$} & \multicolumn{3}{|c|}{ i) Red palm olein } & \multicolumn{3}{|c|}{ ii) Palm olein } \\
\hline & & (a) Deep-fat fryer & (b) Microwave oven & (c) Conventional oven & (a) Deep-fat fryer & (b) Microwave oven & (c) Conventional oven \\
\hline \multirow{13}{*}{ 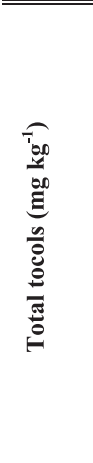 } & $\overline{00}$ & $969.67 \pm 10.21(\mathrm{c})$ & $2070.17 \pm 16.34(\mathrm{c})$ & $939 \pm 10.56(\mathrm{~b}, \mathrm{a})$ & 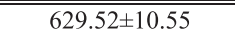 & 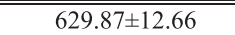 & $635.38 \pm 5.72$ \\
\hline & 15 & $926 \pm 75.39$ & $956.92 \pm 7.09$ & $909.48 \pm 14.01$ & $581.45 \pm 10.25 *(\mathrm{~b}, \mathrm{c})$ & $619.81 \pm 3.27(\mathrm{a}, \mathrm{c})$ & $595.46 \pm 2.85 *(a, b)$ \\
\hline & 30 & $933.54 \pm 64.9(\mathrm{~b})$ & $834.94 \pm 46.26$ (a) & $888.12 \pm 30.04$ & $571.85 \pm 2.95 *$ & $569.17 \pm 4.93 *$ & $582.87 \pm 13.85 *$ \\
\hline & 45 & $917.78 \pm 60.86(b)$ & $669.05 \pm 28.14 *(\mathrm{a}, \mathrm{c})$ & $887.15 \pm 22.93(b)$ & $574.29 \pm 1.53 *(b, c)$ & $545.42 \pm 7.71 *(\mathrm{a}, \mathrm{c})$ & $596.71 \pm 5.35 *(a, b)$ \\
\hline & 60 & $926.06 \pm 109.35(\mathrm{~b})$ & $490.75 \pm 66.73 *(\mathrm{a}, \mathrm{c})$ & $864.03 \pm 11.43(\mathrm{~b})$ & $565.84 \pm 9.71 *$ & $553.37 \pm 8.68 *$ & $568.93 \pm 10.18 *$ \\
\hline & 75 & $891.76 \pm 87.97(\mathrm{~b})$ & $324.47 \pm 64.02 *(\mathrm{a}, \mathrm{c})$ & $817.68 \pm 20.39 *(b)$ & $548.29 \pm 4.52 *(\mathrm{c})$ & $543.19 \pm 4.34 *(\mathrm{c})$ & $564.42 \pm 2.86 *(a, b)$ \\
\hline & 90 & $893.82 \pm 68.02(\mathrm{~b})$ & $237.26 \pm 72.53 *(\mathrm{a}, \mathrm{c})$ & $824.14 \pm 25.25 *(b)$ & $567.94 \pm 16.49 *$ & $525.2 \pm 3.56 *$ & $564.41 \pm 20.91 *$ \\
\hline & 105 & $938.31 \pm 75.75(b)$ & $236.8 \pm 151.07 *(\mathrm{a}, \mathrm{c})$ & $818.16 \pm 7.5 *(b)$ & $569.46 \pm 5.74 *(b)$ & $524.87 \pm 3.09 *(a, c)$ & $563.4 \pm 2.94 *(b)$ \\
\hline & 120 & $929.75 \pm 11.08(b, c)$ & $194.88 \pm 112.45 *(\mathrm{a}, \mathrm{c})$ & $802.89 \pm 12.55 *(a, b)$ & $580.52 \pm 5.56 *(\mathrm{~b}, \mathrm{c})$ & $503.1 \pm 16.97 *(a, c)$ & $550.16 \pm 14.93 *(a, b)$ \\
\hline & 135 & $834.46 \pm 65.1(\mathrm{~b}, \mathrm{c})$ & $59.09 \pm 3.62 *(\mathrm{a}, \mathrm{c})$ & $793.18 \pm 7.37 *(a, b)$ & $589.48 \pm 23.59 *(\mathrm{~b}, \mathrm{c})$ & $504.12 \pm 8.7 *(\mathrm{a}, \mathrm{c})$ & $545.37 \pm 8.55 *(\mathrm{a}, \mathrm{b})$ \\
\hline & 150 & $892.31 \pm 32.3(\mathrm{~b}, \mathrm{c})$ & $62.83 \pm 17.19 *(a, c)$ & $820.15 \pm 147.66 *(a, b)$ & $576.66 \pm 7.47 *(b, c)$ & $477.9 \pm 9.29 *(\mathrm{a}, \mathrm{c})$ & $548.28 \pm 1.33 *(a, b)$ \\
\hline & 165 & $821.03 \pm 51.49(\mathrm{~b}, \mathrm{c})$ & $45.5 \pm 4.15 *(\mathrm{a}, \mathrm{c})$ & $737.73 \pm 37.69 *(a, b)$ & $572.23 \pm 16 *(\mathrm{~b}, \mathrm{c})$ & $474.12 \pm 5.17 *(\mathrm{a}, \mathrm{c})$ & $537.3 \pm 9.26 *(\mathrm{a}, \mathrm{b})$ \\
\hline & 180 & $811.99 \pm 46.99(b, c)$ & $37.34 \pm 11.01 *(\mathrm{a}, \mathrm{c})$ & $739.39 \pm 20.88 *(a, b)$ & $570.39 \pm 4.14 *(\mathrm{~b}, \mathrm{c})$ & $472.15 \pm 3.05 *(a, c)$ & $507.97 \pm 15.96 *(a, b)$ \\
\hline \multirow{13}{*}{ 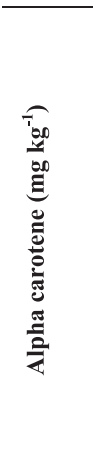 } & $\mathbf{0}$ & $157.74 \pm 10.23(\mathrm{~b}, \mathrm{c})$ & $152.98 \pm 16.38(\mathrm{a})$ & $170.47 \pm 8.87$ (a) & n.d. & n.d. & n.d. \\
\hline & 15 & $121.88 \pm 5.29 *(b, c)$ & $150.39 \pm 11.97(\mathrm{a}, \mathrm{c})$ & $160.96 \pm 2.79(\mathrm{a}, \mathrm{b})$ & n.d. & n.d. & n.d. \\
\hline & 30 & $109.77 \pm 6.09 *(b, c)$ & $138.25 \pm 2.07(\mathrm{a}, \mathrm{c})$ & $153.48 \pm 3.99(\mathrm{a}, \mathrm{b})$ & n.d. & n.d. & n.d. \\
\hline & 45 & $106.55 \pm 10.21 *(b, c)$ & $92.54 \pm 4.08 *(\mathrm{a}, \mathrm{c})$ & $146.80 \pm 2.35 *(a, b)$ & n.d. & n.d. & n.d. \\
\hline & 60 & $92.10 \pm 0.78 *(b, c)$ & $61.92 \pm 9.64 *(a, c)$ & $139.39 \pm 0.53 *(a, b)$ & n.d. & n.d. & n.d. \\
\hline & 75 & $81.96 \pm 3.53 *(b, c)$ & $29.83 \pm 0.71 *(a, c)$ & $130.96 \pm 2.70 *(a, b)$ & n.d. & n.d. & n.d. \\
\hline & 90 & $71.22 \pm 3.24 *(b, c)$ & $15.98 \pm 9.85 *(\mathrm{a}, \mathrm{c})$ & $123.17 \pm 3.24 *(a, b)$ & n.d. & n.d. & n.d. \\
\hline & 105 & $60.99 \pm 1.52 *(b, c)$ & $17.41 \pm 18.70 *(\mathrm{a}, \mathrm{c})$ & $118.64 \pm 11.39 *(a, b)$ & n.d. & n.d. & n.d. \\
\hline & 120 & $57.47 \pm 15.76 *(b, c)$ & $11.35 \pm 11.92 *(\mathrm{a}, \mathrm{c})$ & $107.68 \pm 17.15 *(a, b)$ & n.d. & n.d. & n.d. \\
\hline & 135 & $47.30 \pm 1.20 *(\mathrm{~b}, \mathrm{c})$ & $0.00 \pm 0.00 *(\mathrm{a}, \mathrm{c})$ & $95.71 \pm 10.91 *(a, b)$ & n.d. & n.d. & n.d. \\
\hline & 150 & $40.21 \pm 1.68 *(b, c)$ & $0.00 \pm 0.00 *(a, c)$ & $103.20 \pm 3.17 *(a, b)$ & n.d. & n.d. & n.d. \\
\hline & 165 & $32.61 \pm 4.46 *(\mathrm{~b}, \mathrm{c})$ & $0.00 \pm 0.00 *(a, c)$ & $79.28 \pm 5.22 *(a, b)$ & n.d. & n.d. & n.d. \\
\hline & 180 & $30.38 \pm 2.59 *(b, c)$ & $0.00 \pm 0.00 *(a, c)$ & $78.15 \pm 11.48 *(a, b)$ & n.d. & n.d. & n.d. \\
\hline \multirow{13}{*}{ 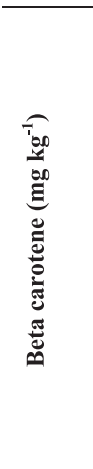 } & 0 & $155.75 \pm 8.79$ & $160.06 \pm 17.93$ & $173.70 \pm 5.68$ & n.d. & n.d. & n.d. \\
\hline & 15 & $108.98 \pm 4.57 *(b, c)$ & $152.30 \pm 10.29$ (a) & $166.43 \pm 6.21(\mathrm{a})$ & n.d. & n.d. & n.d. \\
\hline & 30 & $91.83 \pm 2.35 *(b, c)$ & $134.28 \pm 10.33 *(a, c)$ & $153.73 \pm 5.14(\mathrm{a}, \mathrm{b})$ & n.d. & n.d. & n.d. \\
\hline & 45 & $77.40 \pm 3.22 *(\mathrm{c})$ & $82.18 \pm 5.00 *(\mathrm{c})$ & $144.96 \pm 1.76 *(a, b)$ & n.d. & n.d. & n.d. \\
\hline & 60 & $68.12 \pm 0.31 *(b, c)$ & $49.48 \pm 10.75 *(\mathrm{a}, \mathrm{c})$ & $135.56 \pm 1.31 *(a, b)$ & n.d. & n.d. & n.d. \\
\hline & 75 & $59.56 \pm 1.87 *(\mathrm{~b}, \mathrm{c})$ & $20.70 \pm 0.09 *(\mathrm{a}, \mathrm{c})$ & $125.16 \pm 4.47 *(a, b)$ & n.d. & n.d. & n.d. \\
\hline & 90 & $50.24 \pm 1.76 *(\mathrm{~b}, \mathrm{c})$ & $10.57 \pm 6.85 *(\mathrm{a}, \mathrm{c})$ & $125.39 \pm 7.46 *(\mathrm{a}, \mathrm{b})$ & n.d. & n.d. & n.d. \\
\hline & 105 & $42.23 \pm 1.76 *(b, c)$ & $12.25 \pm 12.85 *(\mathrm{a}, \mathrm{c})$ & $109.44 \pm 10.66 *(a, b)$ & n.d. & n.d. & n.d. \\
\hline & 120 & $43.64 \pm 4.62 *(b, c)$ & $7.09 \pm 8.19 *(a, c)$ & $123.47 \pm 11.17 *(a, b)$ & n.d. & n.d. & n.d. \\
\hline & 135 & $31.39 \pm 0.93 *(b, c)$ & $0.00 \pm 0.00 *(a, c)$ & $105.40 \pm 15.97 *(a, b)$ & n.d. & n.d. & n.d. \\
\hline & 150 & $26.37 \pm 1.46 *(\mathrm{~b}, \mathrm{c})$ & $0.00 \pm 0.00 *(a, c)$ & $85.59 \pm 7.48 *(a, b)$ & n.d. & n.d. & n.d. \\
\hline & 165 & $20.60 \pm 3.46 *(b, c)$ & $0.00 \pm 0.00 *(\mathrm{a}, \mathrm{c})$ & $67.69 \pm 4.87 *(a, b)$ & n.d. & n.d. & n.d. \\
\hline & 180 & $18.97 \pm 2.02 *(\mathrm{~b}, \mathrm{c})$ & $0.00 \pm 0.00 *(a, c)$ & $68.80 \pm 8.52 *(a, b)$ & n.d. & n.d. & n.d. \\
\hline
\end{tabular}

Values are expressed as mean \pm SD (replicate analysis x 2 experiments). Data were measured by One-way Anova with Tukey posthoc multiple comparison tests to assess the differences between groups; $*(p<0.05)$ compared to 0 minutes within group. Mean values followed by different letters $(a, b, c)$ are significant different $(p<0.05)$ between groups for different heating technique at same time point. n.d. (not detectable).

formed had been reported ${ }^{14,21-27)}$. In contrary to the current study, Mahmoud et al. and Caponio et al. reported that microwave and conventional oven heating had no difference in terms of oxidation products ${ }^{28,29}$. However, it is a point to note that duration of heating for both studies was rather short (30 minutes) to exhibit an effect. In accordance to the current observation, Poiana reported a higher oxidation degree by microwave oven as compared to conventional heating upon longer heating period (240 minutes $)^{26)}$.

FFA is one of the most important indicators of oil deteri- oration during heating. The formation of FFA is due to the hydrolysis of triacylglycerol and hydroperoxide decomposition in oil during heating in the presence of moisture and $\mathrm{air}^{30)}$. According to Malaysian Standard for Palm Oil Specifications, FFA (as palmitic) content of refined oils should be less than $0.1 \%{ }^{31}$. The levels in initial fresh test oil were $0.06 \pm 0.01 \%$ in palm olein and $0.07 \pm 0.01 \%$ in red palm olein (Table 2). The United States Department of Agriculture (USDA) requires the frying oils to be discarded when the free fatty acid (FFA) content in the oil exceeds $2 \%{ }^{18}$. A 
R. Loganathan, A. H. Ahmad Tarmizi, S. R. Vethakkan et al.

Table 2 Changes in PV, AnV, TOTOX and FFA upon heating.

\begin{tabular}{|c|c|c|c|c|c|c|c|}
\hline \multirow{2}{*}{ Parameter } & \multirow{2}{*}{ Time } & \multicolumn{3}{|c|}{ i) Red palm olein } & \multicolumn{3}{|c|}{ ii) Palm Olein } \\
\hline & & (a) Deep Fryer & (b)Microwave & (c) Oven & (a) Deep Fryer & (b)Microwave & (c) Oven \\
\hline \multirow{13}{*}{$\begin{array}{l}\text { PV } \\
\left(\text { meq } \mathrm{O}_{2} \mathrm{~kg}^{-1}\right)\end{array}$} & 0 & $0.33 \pm 0.05$ & $0.33 \pm 0.08$ & $0.33 \pm 0.05$ & $0.43 \pm 0.05$ & $0.38 \pm 0.08$ & $0.40 \pm 0.06$ \\
\hline & 15 & $1.20 \pm 0.09(\mathrm{~b})$ & $1.73 \pm 0.18 *(\mathrm{a}, \mathrm{c})$ & $1.00 \pm 0.14 *(b)$ & $0.38 \pm 0.08(\mathrm{~b}, \mathrm{c})$ & $1.46 \pm 0.17 *(\mathrm{a}, \mathrm{c})$ & $0.90 \pm 0.09 *(a, b)$ \\
\hline & 30 & $1.50 \pm 1.14(\mathrm{~b})$ & $2.05 \pm 0.10 *(\mathrm{a}, \mathrm{c})$ & $1.53 \pm 0.08 *(b)$ & $0.45 \pm 0.05(\mathrm{~b}, \mathrm{c})$ & $1.55 \pm 0.22 *(a)$ & $1.47 \pm 0.08 *(a)$ \\
\hline & 45 & $1.65 \pm 0.10(b)$ & $2.81 \pm 0.22 *(\mathrm{a}, \mathrm{c})$ & $2.03 \pm 0.10 *(a, b)$ & $0.48 \pm 0.08(\mathrm{~b}, \mathrm{c})$ & $1.68 \pm 0.17 *(\mathrm{a}, \mathrm{c})$ & $1.86 \pm 0.08 *(\mathrm{a}, \mathrm{b})$ \\
\hline & 60 & $1.76 \pm 0.08(\mathrm{~b}, \mathrm{c})$ & $3.99 \pm 0.14 *(\mathrm{a}, \mathrm{c})$ & $2.26 \pm 0.12 *(a, b)$ & $0.57 \pm 0.14(\mathrm{~b}, \mathrm{c})$ & $1.86 \pm 0.15 *(a)$ & $1.93 \pm 0.10 *(\mathrm{a})$ \\
\hline & 75 & $1.06 \pm 0.08(\mathrm{~b}, \mathrm{c})$ & $5.46 \pm 0.17 *(\mathrm{a}, \mathrm{c})$ & $2.75 \pm 0.10 *(a, b)$ & $0.60 \pm 0.13(b, c)$ & $2.06 \pm 0.20 *(a)$ & $1.95 \pm 0.08 *(a)$ \\
\hline & 90 & $2.06 \pm 0.08 *(b, c)$ & $5.89 \pm 0.20 *(\mathrm{a}, \mathrm{c})$ & $2.83 \pm 0.16 *(a, b)$ & $0.63 \pm 0.05 *(b, c)$ & $2.33 \pm 0.23 *(a)$ & $2.10 \pm 0.13 *(a)$ \\
\hline & 105 & $2.11 \pm 0.04 *(b, c)$ & $6.44 \pm 0.17 *(\mathrm{a}, \mathrm{c})$ & $3.75 \pm 0.15 *(a, b)$ & $0.69 \pm 0.14 *(b, c)$ & $2.88 \pm 0.15 *(\mathrm{a}, \mathrm{c})$ & $4.30 \pm 0.21 *(a, b)$ \\
\hline & 120 & $2.21 \pm 0.04 *(b, c)$ & $6.64 \pm 0.18 *(\mathrm{a}, \mathrm{c})$ & $4.01 \pm 0.22 *(a, b)$ & $0.70 \pm 0.11 *(\mathrm{~b}, \mathrm{c})$ & $4.38 \pm 0.36 *(\mathrm{a}, \mathrm{c})$ & $5.78 \pm 0.10 *(a, b)$ \\
\hline & 135 & $2.26 \pm 0.05 *(\mathrm{~b}, \mathrm{c})$ & $7.45 \pm 0.42 *(\mathrm{a}, \mathrm{c})$ & $4.06 \pm 0.12 *(a, b)$ & $0.72 \pm 0.08 *(\mathrm{~b}, \mathrm{c})$ & $5.46 \pm 0.24 *(\mathrm{a}, \mathrm{c})$ & $6.18 \pm 0.13 *(a, b)$ \\
\hline & 150 & $2.36 \pm 0.10 *(b, c)$ & $8.45 \pm 0.28 *(\mathrm{a}, \mathrm{c})$ & $4.25 \pm 0.17 *(a, b)$ & $0.85 \pm 0.11 *(b, c)$ & $5.96 \pm 0.32 *(\mathrm{a}, \mathrm{c})$ & $6.36 \pm 0.10 *(a, b)$ \\
\hline & 165 & $2.55 \pm 0.18 *(b, c)$ & $9.34 \pm 0.13 *(\mathrm{a}, \mathrm{c})$ & $4.86 \pm 0.16 *(a, b)$ & $1.47 \pm 0.12 *(b, c)$ & $6.47 \pm 0.38 *(a)$ & $6.71 \pm 0.22 *(a)$ \\
\hline & 180 & $3.13 \pm 0.08 *(b, c)$ & $11.40 \pm 0.68 *(a, c)$ & $5.54 \pm 0.08 *(\mathrm{a}, \mathrm{b})$ & $2.60 \pm 0.36 *(\mathrm{~b}, \mathrm{c})$ & $6.87 \pm 0.29 *(a)$ & $6.84 \pm 0.15 *(a)$ \\
\hline \multirow[t]{13}{*}{ AnV (unit) } & 0 & 0 & 1.62 & 1.58 & 1. & 0 & 1.1 \\
\hline & 15 & $10.62 \pm 0.50(b, c)$ & $3.68 \pm 0.34 *(a, c)$ & $2.18 \pm 0.13(\mathrm{a}, \mathrm{b})$ & $9.09 \pm 1.10 *(b, c)$ & $1.99 \pm 0.27(\mathrm{a}, \mathrm{c})$ & $1.46 \pm 0.17(\mathrm{a}, \mathrm{b})$ \\
\hline & 30 & $11.50 \pm 1.92 *(b, c)$ & $6.72 \pm 0.53 *(a, c)$ & $2.31 \pm 0.24(\mathrm{a}, \mathrm{b})$ & $9.41 \pm 1.02 *(b, c)$ & $3.96 \pm 0.07 *(a, c)$ & $2.21 \pm 0.13 *(\mathrm{a}, \mathrm{b})$ \\
\hline & 45 & $13.22 \pm 1.23 *(\mathrm{~b}, \mathrm{c})$ & $7.05 \pm 1.00 *(\mathrm{a}, \mathrm{c})$ & $2.49 \pm 0.26(\mathrm{a}, \mathrm{b})$ & $10.07 \pm 0.94 *(\mathrm{~b}, \mathrm{c})$ & $12.37 \pm 1.98 *(\mathrm{a}, \mathrm{c})$ & $2.33 \pm 0.32 *(a, b)$ \\
\hline & 60 & $15.12 \pm 1.66 *(b, c)$ & $11.58 \pm 0.64 *(\mathrm{a}, \mathrm{c})$ & $2.55 \pm 0.20 *(a, b)$ & $10.21 \pm 1.06 *(b, c)$ & $14.47 \pm 1.07 *(\mathrm{a}, \mathrm{c})$ & $3.90 \pm 0.19 *(a, b)$ \\
\hline & 75 & $16.56 \pm 0.44 *(\mathrm{~b}, \mathrm{c})$ & $13.05 \pm 0.97 *(\mathrm{a}, \mathrm{c})$ & $2.69 \pm 0.39 *(\mathrm{a}, \mathrm{b})$ & $11.09 \pm 1.00 *(\mathrm{~b}, \mathrm{c})$ & $15.34 \pm 0.75 *(\mathrm{a}, \mathrm{c})$ & $4.00 \pm 0.11 *(a, b)$ \\
\hline & 90 & $18.38 \pm 1.15 *(\mathrm{~b}, \mathrm{c})$ & $16.13 \pm 0.52 *(a, c)$ & $2.89 \pm 0.18 *(a, b)$ & $12.00 \pm 1.44 *(b, c)$ & $16.36 \pm 0.99 *(\mathrm{a}, \mathrm{c})$ & $4.00 \pm 0.09 *(a, b)$ \\
\hline & 105 & $19.58 \pm 1.30 *(b, c)$ & $17.66 \pm 1.22 *(\mathrm{a}, \mathrm{c})$ & $3.48 \pm 0.42 *(a, b)$ & $12.11 \pm 1.50 *(\mathrm{~b}, \mathrm{c})$ & $18.66 \pm 1.70 *(\mathrm{a}, \mathrm{c})$ & $5.35 \pm 0.31 *(a, b)$ \\
\hline & 120 & $20.53 \pm 1.54 *(\mathrm{c})$ & $19.52 \pm 0.97 *(\mathrm{c})$ & $5.63 \pm 0.26 *(a, b)$ & $12.38 \pm 1.17 *(\mathrm{~b}, \mathrm{c})$ & $20.05 \pm 0.68 *(\mathrm{a}, \mathrm{c})$ & $6.47 \pm 0.67 *(\mathrm{a}, \mathrm{b})$ \\
\hline & 135 & $22.18 \pm 1.90 *(\mathrm{c})$ & $21.50 \pm 1.59 *(\mathrm{c})$ & $7.11 \pm 0.36 *(a, b)$ & $13.06 \pm 1.03 *(b, c)$ & $20.16 \pm 0.81 *(\mathrm{a}, \mathrm{c})$ & $6.70 \pm 0.15 *(a, b)$ \\
\hline & 150 & $22.90 \pm 1.86 *(\mathrm{c})$ & $22.63 \pm 0.96 *(\mathrm{c})$ & $7.43 \pm 0.44 *(a, b)$ & $13.31 \pm 1.57 *(b, c)$ & $21.25 \pm 0.79 *(a, c)$ & $6.98 \pm 0.61 *(a, b)$ \\
\hline & 165 & $24.28 \pm 2.26 *(\mathrm{c})$ & $25.24 \pm 1.25 *(\mathrm{c})$ & $9.19 \pm 0.55 *(a, b)$ & $13.47 \pm 1.25 *(\mathrm{~b}, \mathrm{c})$ & $22.59 \pm 1.03 *(\mathrm{a}, \mathrm{c})$ & $7.15 \pm 0.17 *(a, b)$ \\
\hline & 180 & $24.53 \pm 1.12 *(\mathrm{c})$ & $25.88 \pm 0.83 *(\mathrm{c})$ & $14.24 \pm 1.31 *(\mathrm{a}, \mathrm{b})$ & $13.75 \pm 1.41 *(\mathrm{~b}, \mathrm{c})$ & $23.73 \pm 0.33 *(\mathrm{a}, \mathrm{c})$ & $8.41 \pm 0.31 *(a, b)$ \\
\hline \multirow{13}{*}{$\begin{array}{l}\text { TOTOX } \\
\text { (unit) }\end{array}$} & 0 & $2.22=$ & $2.28 \pm 0.18$ & 0.13 & $1.91=$ & $1.71 \pm 0.18$ & .26 \\
\hline & 15 & $13.01 \pm 0.57 *(\mathrm{~b}, \mathrm{c})$ & $14 \pm 0.67 *(\mathrm{a}, \mathrm{c})$ & $4.18 \pm 0.24 *(a, b)$ & $9.85 \pm 1.1$ & $4.92 \pm 0.42 *(\mathrm{a}, \mathrm{c})$ & $*(a, b)$ \\
\hline & 30 & $14.49 \pm 1.76 *(\mathrm{~b}, \mathrm{c})$ & $10.81 \pm 0.61 *(\mathrm{a}, \mathrm{c})$ & $5.37 \pm 0.16 *(a, b)$ & $10.31 \pm 1.03 *(\mathrm{~b}, \mathrm{c})$ & $7.05 \pm 0.50 *(\mathrm{a}, \mathrm{c})$ & $5.15 \pm 0.27 *(\mathrm{a}, \mathrm{b})$ \\
\hline & 45 & $16.51 \pm 1.37 *(b, c)$ & $12.67 \pm 1.34 *(\mathrm{a}, \mathrm{c})$ & $6.55 \pm 0.27 *(a, b)$ & $11.04 \pm 1.02 *(b, c)$ & $15.73 \pm 1.82 *(\mathrm{a}, \mathrm{c})$ & $6.06 \pm 0.42 *(a, b)$ \\
\hline & 60 & $18.65 \pm 1.78 *(\mathrm{~b}, \mathrm{c})$ & $19.56 \pm 0.70 *(\mathrm{a}, \mathrm{c})$ & $7.08 \pm 0.35 *(a, b)$ & $11.34 \pm 1.17 *(\mathrm{~b}, \mathrm{c})$ & $18.20 \pm 1.17 *(a, c)$ & $7.77 \pm 0.28 *(\mathrm{a}, \mathrm{b})$ \\
\hline & 75 & $20.49 \pm 0.49 *(\mathrm{~b}, \mathrm{c})$ & $23.97 \pm 1.24 *(\mathrm{a}, \mathrm{c})$ & $8.19 \pm 0.46 *(a, b)$ & $12.28 \pm 1.12 *(\mathrm{~b}, \mathrm{c})$ & $19.47 \pm 0.97 *(\mathrm{a}, \mathrm{c})$ & $7.89 \pm 0.27 *(a, b)$ \\
\hline & 90 & $22.51 \pm 1.26 *(\mathrm{~b}, \mathrm{c})$ & $27.92 \pm 0.87 *(\mathrm{a}, \mathrm{c})$ & $8.55 \pm 0.35 *(a, b)$ & $13.26 \pm 1.45 *(b, c)$ & $21.02 \pm 1.25 *(\mathrm{a}, \mathrm{c})$ & $8.20 \pm 0.21 *(a, b)$ \\
\hline & 105 & $23.81 \pm 1.33 *(b, c)$ & $30.54 \pm 1.47 *(\mathrm{a}, \mathrm{c})$ & $10.98 \pm 0.55 *(\mathrm{a}, \mathrm{b})$ & $13.49 \pm 1.60 *(b, c)$ & $24.42 \pm 1.72 *(a, c)$ & $13.94 \pm 0.30 *(\mathrm{a}, \mathrm{b})$ \\
\hline & 120 & $24.96 \pm 1.49 *(\mathrm{~b}, \mathrm{c})$ & $32.79 \pm 1.12 *(\mathrm{a}, \mathrm{c})$ & $13.65 \pm 0.47 *(a, b)$ & $13.78 \pm 1.24 *(\mathrm{~b}, \mathrm{c})$ & $28.81 \pm 0.53 *(\mathrm{a}, \mathrm{c})$ & $18.03 \pm 0.71 *(a, b)$ \\
\hline & 135 & $26.71 \pm 1.92 *(b, c)$ & $36.40 \pm 1.03 *(a, c)$ & $15.23 \pm 0.40 *(a, b)$ & $14.49 \pm 1.14 *(b, c)$ & $31.08 \pm 1.06 *(\mathrm{a}, \mathrm{c})$ & $19.06 \pm 0.37 *(a, b)$ \\
\hline & 150 & $27.63 \pm 1.86 *(\mathrm{~b}, \mathrm{c})$ & $39.54 \pm 0.98 *(\mathrm{a}, \mathrm{c})$ & $15.92 \pm 0.65 *(\mathrm{a}, \mathrm{b})$ & $15.00 \pm 1.55 *(\mathrm{~b}, \mathrm{c})$ & $33.16 \pm 1.35 *(\mathrm{a}, \mathrm{c})$ & $19.70 \pm 0.76 *(a, b)$ \\
\hline & 165 & $29.38 \pm 2.21 *(\mathrm{~b}, \mathrm{c})$ & $43.92 \pm 1.40 *(a, c)$ & $18.91 \pm 0.75 *(a, b)$ & $16.40 \pm 1.33 *(b, c)$ & $35.54 \pm 1.68 *(\mathrm{a}, \mathrm{c})$ & $20.57 \pm 0.57 *(a, b)$ \\
\hline & 180 & $30.79 \pm 1.08 *(b, c)$ & $48.68 \pm 1.71 *(a, c)$ & $25.32 \pm 1.41 *(\mathrm{a}, \mathrm{b})$ & $18.95 \pm 2.08 *(\mathrm{~b}, \mathrm{c})$ & $37.48 \pm 0.72 *(a, c)$ & $22.09 \pm 0.46 *(a, b)$ \\
\hline \multirow[t]{13}{*}{ FFA $(\%)$} & 0 & 0.07 & 0.07 & 0.0 & 0.06 & $0.05 \pm 0$ & 0.06 \\
\hline & 15 & $0.07 \pm 0.00$ & $0.07 \pm 0.00$ & $0.07 \pm 0.00$ & $0.05 \pm 0.00(\mathrm{c})$ & $0.05 \pm 0.00(\mathrm{c})$ & $0.06 \pm 0.00(\mathrm{~b}, \mathrm{a})$ \\
\hline & 30 & $0.07 \pm 0.00$ & $0.07 \pm 0.00$ & $0.07 \pm 0.00$ & $0.07 \pm 0.01 *(\mathrm{~b})$ & $0.05 \pm 0.00(\mathrm{a}, \mathrm{c})$ & $0.06 \pm 0.00 *(\mathrm{~b})$ \\
\hline & 45 & $0.07 \pm 0.01$ & $0.07 \pm 0.00$ & $0.07 \pm 0.00$ & $0.08 \pm 0.00 *(\mathrm{~b}, \mathrm{c})$ & $0.05 \pm 0.00(\mathrm{a}, \mathrm{c})$ & $0.06 \pm 0.00 *(\mathrm{a}, \mathrm{b})$ \\
\hline & 60 & $0.07 \pm 0.00$ & $0.07 \pm 0.00$ & $0.07 \pm 0.00$ & $0.09 \pm 0.00 *(\mathrm{~b}, \mathrm{c})$ & $0.05 \pm 0.00(\mathrm{a}, \mathrm{c})$ & $0.06 \pm 0.00 *(\mathrm{a}, \mathrm{b})$ \\
\hline & 75 & $0.07 \pm 0.00(\mathrm{c})$ & $0.07 \pm 0.00(\mathrm{c})$ & $0.08 \pm 0.00 *(a, b)$ & $0.09 \pm 0.00 *(\mathrm{~b}, \mathrm{c})$ & $0.06 \pm 0.00 *(a, c)$ & $0.07 \pm 0.00 *(\mathrm{a}, \mathrm{b})$ \\
\hline & 90 & $0.07 \pm 0.00(\mathrm{c})$ & $0.07 \pm 0.00$ & $0.08 \pm 0.01 *(\mathrm{a})$ & $0.09 \pm 0.00 *(\mathrm{~b}, \mathrm{c})$ & $0.06 \pm 0.00 *(\mathrm{a}, \mathrm{c})$ & $0.07 \pm 0.00 *(\mathrm{a}, \mathrm{b})$ \\
\hline & 105 & $0.07 \pm 0.00(\mathrm{c})$ & $0.07 \pm 0.009(\mathrm{c})$ & $0.08 \pm 0.00 *(\mathrm{a}, \mathrm{b})$ & $0.10 \pm 0.00 *(\mathrm{~b}, \mathrm{c})$ & $0.06 \pm 0.00 *(\mathrm{a}, \mathrm{c})$ & $0.07 \pm 0.00 *(\mathrm{a}, \mathrm{b})$ \\
\hline & 120 & $0.07 \pm 0.00(\mathrm{c})$ & $0.07 \pm 0.00(\mathrm{c})$ & $0.09 \pm 0.00 *(a, b)$ & $0.10 \pm 0.00 *(b, c)$ & $0.06 \pm 0.01 *(\mathrm{a}, \mathrm{c})$ & $0.07 \pm 0.00 *(a, b)$ \\
\hline & 135 & $0.07 \pm 0.00(\mathrm{c})$ & $0.07 \pm 0.00(\mathrm{c})$ & $0.09 \pm 0.00 *(\mathrm{a}, \mathrm{b})$ & $0.10 \pm 0.00 *(\mathrm{~b}, \mathrm{c})$ & $0.07 \pm 0.01 *(\mathrm{a}, \mathrm{c})$ & $0.08 \pm 0.00 *(\mathrm{a}, \mathrm{b})$ \\
\hline & 150 & $0.07 \pm 0.00(\mathrm{c})$ & $0.08 \pm 0.00 *(\mathrm{c})$ & $0.09 \pm 0.00 *(a, b)$ & $0.10 \pm 0.00 *(b, c)$ & $0.07 \pm 0.01 *(\mathrm{a}, \mathrm{c})$ & $0.08 \pm 0.00 *(a, b)$ \\
\hline & 165 & $0.07 \pm 0.00(\mathrm{~b}, \mathrm{c})$ & $0.09 \pm 0.00 *(\mathrm{a})$ & $0.09 \pm 0.00 *(a)$ & $0.10 \pm 0.00 *(b, c)$ & $0.07 \pm 0.01 *(\mathrm{a}, \mathrm{c})$ & $0.08 \pm 0.00 *(\mathrm{a}, \mathrm{b})$ \\
\hline & 180 & $0.07 \pm 0.00(\mathrm{~b}, \mathrm{c})$ & $0.09 \pm 0.00 *(\mathrm{a}, \mathrm{c})$ & $0.09 \pm 0.00 *(\mathrm{a}, \mathrm{b})$ & $0.11 \pm 0.00 *(\mathrm{~b}, \mathrm{c})$ & $0.08 \pm 0.01 *(\mathrm{a}, \mathrm{c})$ & $0.09 \pm 0.00 *(\mathrm{a}, \mathrm{b})$ \\
\hline
\end{tabular}

Values are expressed as mean $\pm \mathrm{SD}$ (Triplicate analysis $\mathrm{x} 2$ experiments). Data were measured by One-way Anova with Tukey posthoc multiple comparison tests to assess the differences between groups; $*(p<0.05)$ compared to 0 minutes within group. Mean values followed by different letters (a,b,c) are significant different $(p<0.05)$ between groups for different heating technique at same time point. 
desirable FFA level in fresh frying oil is $0.03 \%$ to $0.05 \%$. The FFA content in absorbed frying oils used for snack food products ranges from $0.25 \%$ to $0.40 \%$. A portion of frying oil will be replaced with fresh oil once the FFA level exceeds $0.35 \%{ }^{18)}$. It is a standard procedure for established snack food industry to discard the frying oil once the FFA level reaches end point. The end point of FFA varies depending on type of fried food eg. 2 to $2.5 \%$ for breaded and coated food, $0.5 \%$ for instant noodles and potato chips whereas $1 \%$ is applied for pre-fried French fries ${ }^{32}$. In our study, the FFA contents significantly increased time dependently with heating at $180^{\circ} \mathrm{C}$, however the increase was very small and was within regulatory guidelines. No significant difference $(p<0.05)$ in FFA levels were found throughout the heating period with red palm olein for deep-fat fryer(Table 2). Hence in the current study, no major hydrolytic degradation was observed with regards to FFA content in both test fats studied. Similar to current study, heating oil itself at $180^{\circ} \mathrm{C}$ did not cause major triacylglycerol hydrolysis ${ }^{14,22,23,27,33,34)}$. However, in the presence of food matrix, the acidity values rapidly increased probably due to lipases reaction ${ }^{6,35-40)}$.

No trans oleic and linoleic acid were found in the fresh and heated oils. The degree of oxidative deterioration parameter, the ratio of $\mathrm{C} 18: 2 / \mathrm{C} 16: 0$ also remained stable throughout the heating period for all three tested cooking techniques (Table 3). Palm olein blended with canola oil was found to show better oxidative stability based on C18:2/C16:0 than pure canola oil upon microwave oven heating ${ }^{27)}$. Most papers on cooking oils have reported a reduction in unsaturated fatty acids (MUFA and PUFA) ${ }^{24,27,35)}$ and increase in trans-fat content ${ }^{18,35)}$ upon prolonged heating.

Under similar oil heating conditions with conventional heating (by deep-fat fryer and hot plate), microwave oven and conventional oven; a similar trend of increase in hydroperoxides as secondary oxidation products are formed had been reported by other groups in several oils: palm olein ${ }^{41}$, sunflower oil ${ }^{25,41}$, palm olein and sunflower oil blend ${ }^{24,25,41,42)}$, extra virgin olive oil ${ }^{14,21)}$, virgin olive oil ${ }^{14,22,23)}$, pork lard, rapeseed oil, groundnut oil ${ }^{25)}$, canola oil ${ }^{24)}$, grape seed oil ${ }^{42)}$. Contrasting with our current study, Mahmoud et al. and Caponio et al. reported that there was no difference between microwave and conventional oven heating in terms of oxidation products in virgin olive oil ${ }^{28,29)}$, sunflower oil ${ }^{29)}$ and peanut oil ${ }^{29)}$. However, it is important to note that duration of heating for both of the afore mentioned studies was rather too short (30 minutes) to exhibit an effect. We managed to observe significant changes for PV, AnV and TOTOX at 15 min with all the heating techniques, but changes in microwave oven and conventional oven heating were only evident after $45 \mathrm{~min}$ for tocotrienols and 75 min for FFA for antioxidant rich red palm olein. In accordance with our current observations, Poiana also re- ported a higher degree of oxidation with the microwave oven as compared to conventional heating upon longer heating periods $(240 \mathrm{~min})^{26)}$.

Positive correlation was observed within the groups of phytonutrients (Tables 4 and $5(\mathrm{a})$ ) and physico-chemical (Tables 4 and 5(b)) parameters interactions respectively. Protective role of phytonutrients on physico-chemical parameters were shown with strong negative correlation on both oils tested (Table 4(c)). Phytonutrient-rich red palm olein exhibited stronger correlation on interaction "between phytonutrients" (Table 4(a)) and "between phytonutrients and physico-chemical parameters" (Table 4 (c) ) respectively as compared to palm olein (Tables 5 (a) and (c)). Microwave oven had the strongest association between the parameters, followed by conventional oven and lastly deep-fat fryer (medium correlation). This implies that phytonutrients could be retained better and physicochemical parameters was least affected with deep-fat fryer technique, followed by conventional oven and lastly microwave oven.

The fact that PV, AnV, TOTOX and FFA values measured in red palm oil appeared higher than that in palm oil was mainly due to the presence and interference of carotenoid pigments in the colorimetric method employed ${ }^{43}$. In addition, we did not include food matrix and replenish the oil during heating procedure as we intended to check retention of tocols and carotenes upon heating.

\section{Conclusion}

Based on the present study, it can be concluded that red palm olein and palm olein possess good thermal stability based on their low rate of hydrolytic and oxidative degradation. Phytonutrients particularly tocols are better retained when heat transfer is done by convection cooking i.e. deep-fat fryer and conventional oven heating for both oils. However, red palm olein may not be suitable for longer heating processes when carotene retention is of concern. Microwave heating influenced the stability of phytonutrients in red palm olein adversely.

\section{Acknowledgments}

The authors thank the Director-General of the Malaysian Palm Oil Board for permission to publish this study. Sincere acknowledgements for technical support from Ghazali Abd Razak, Dr Fu Ju Yen and Puvaneswari Meganathan. We thank research assistants Fatmawati Othman and Noraqmar Shafie; and practical trainees, Nor Hafizah Md Said, Azrul Arif Mohd Said, Samantha Ling Yuan Ci and Shalini Selvarajah. 

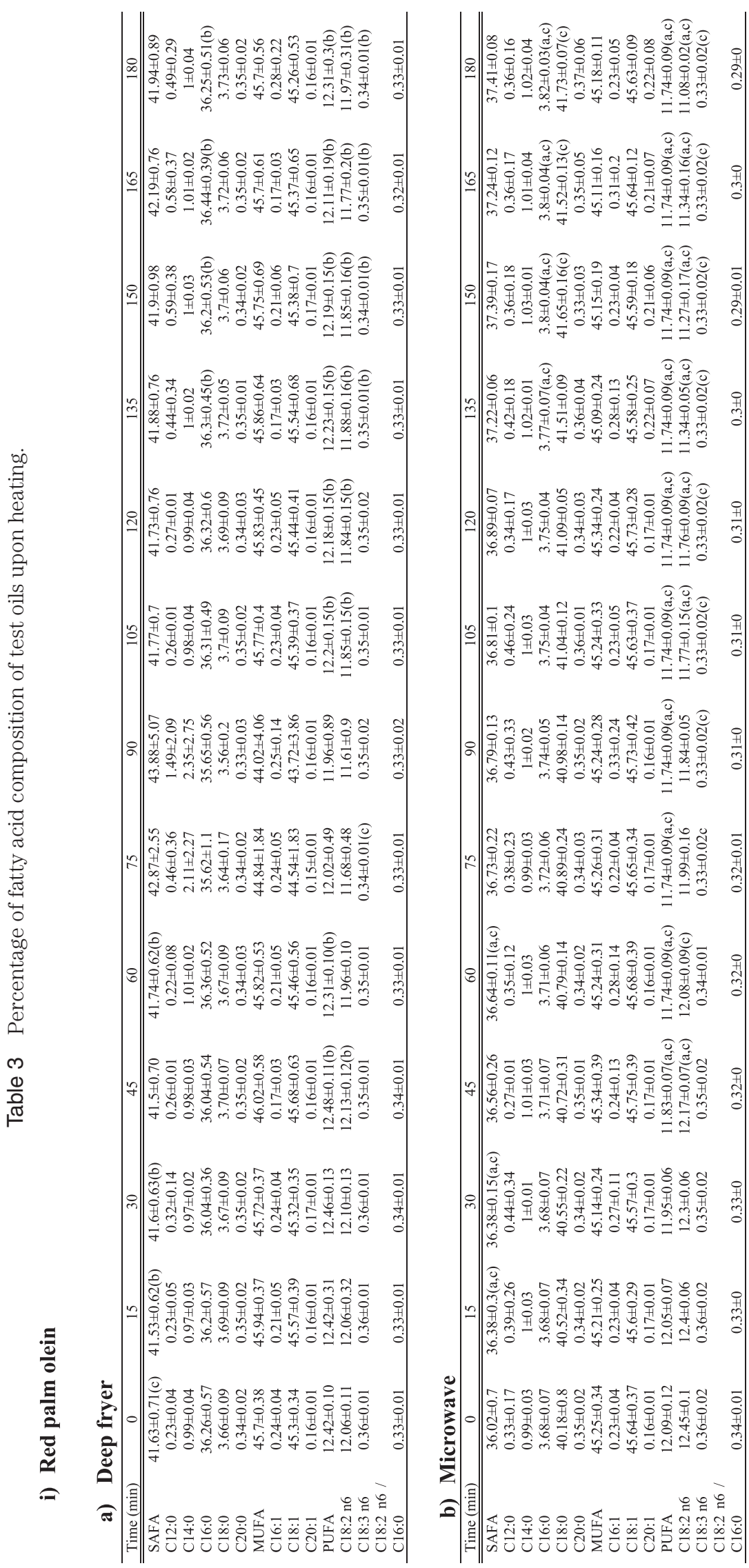


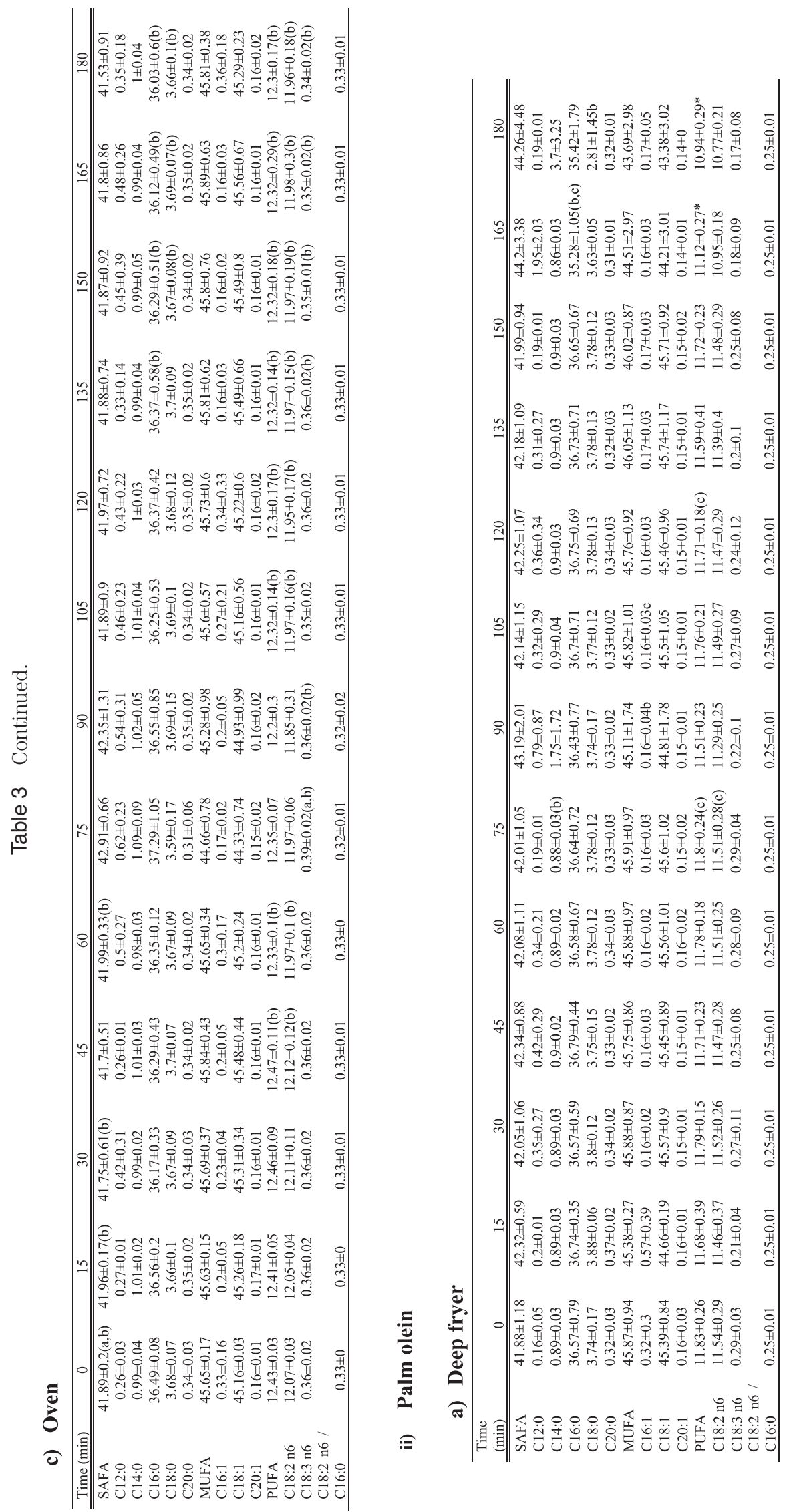



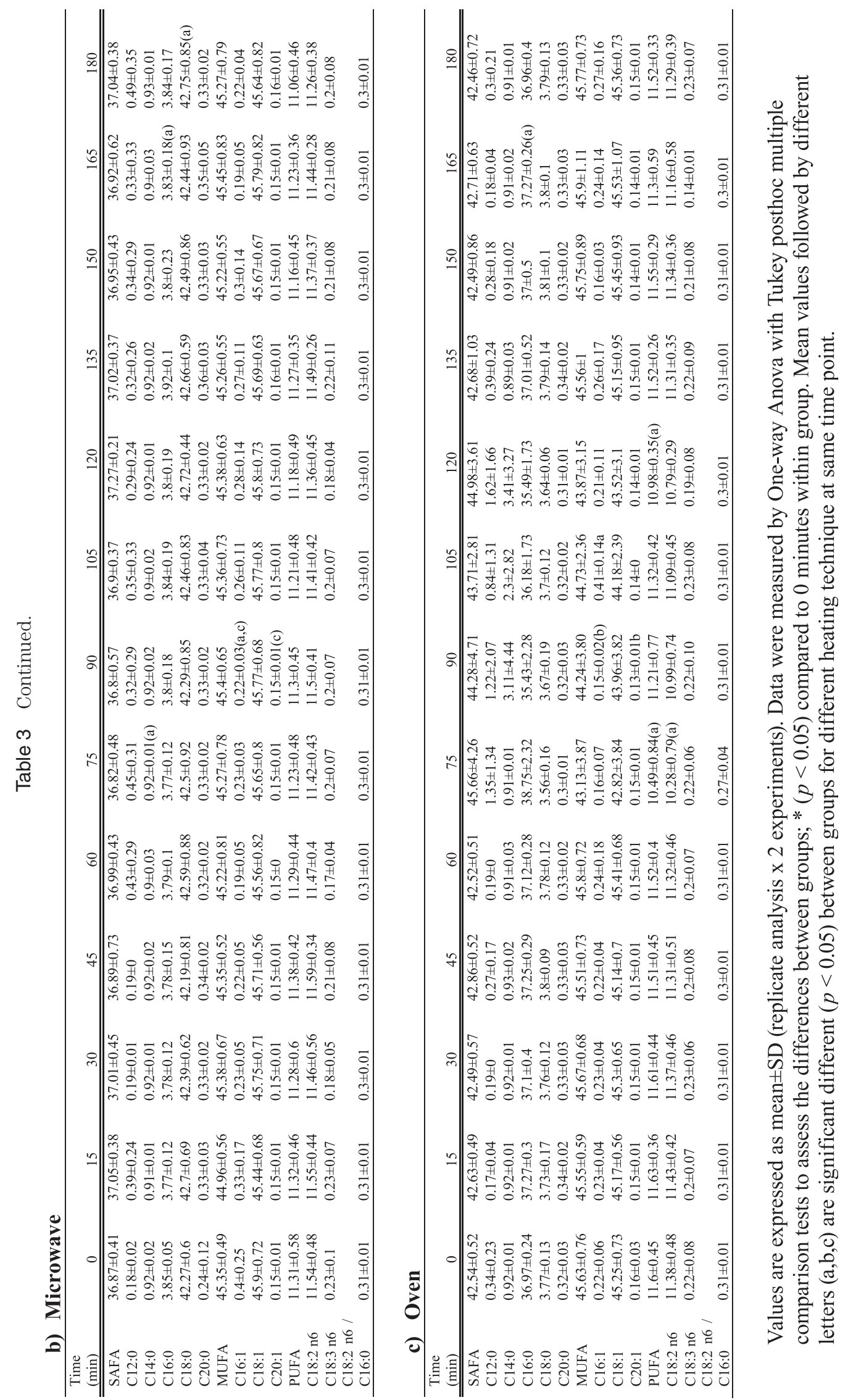
ถู

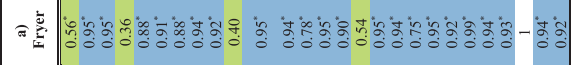

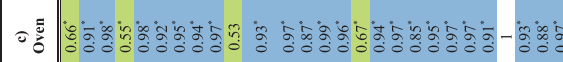

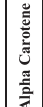

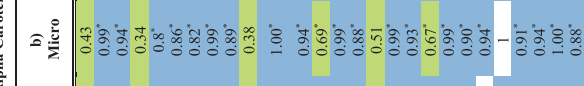
。ำ

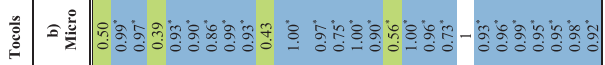
7. ๑ำ

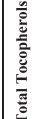
4. on - จำ | 홍 ค는 ๑ำด | 厗 존 - จด โลำ 1. roำ ํำ 耪

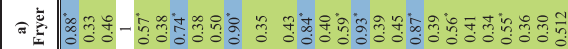
จำ

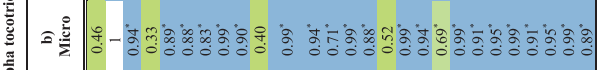
I

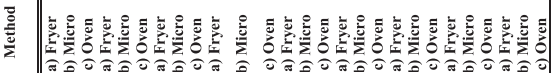

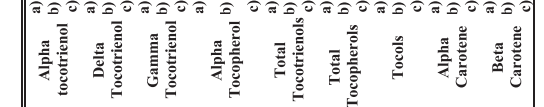

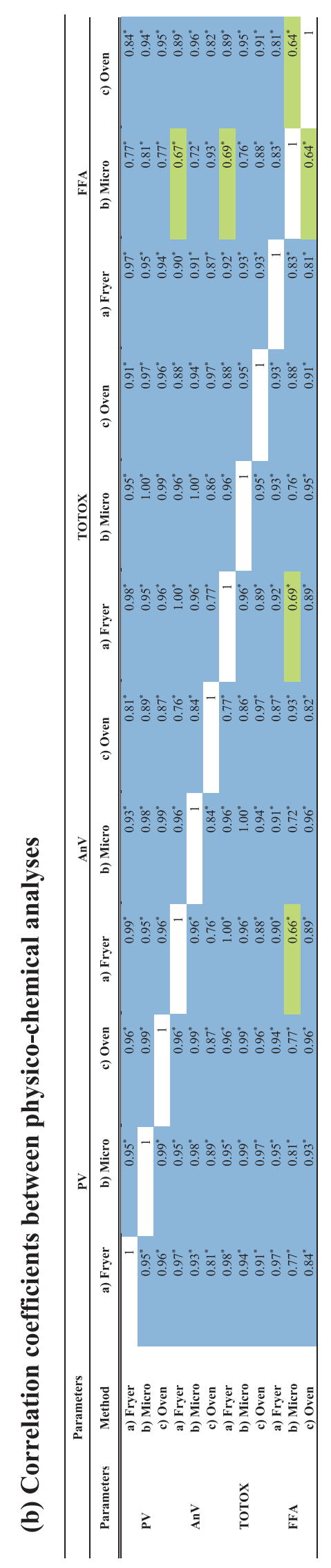

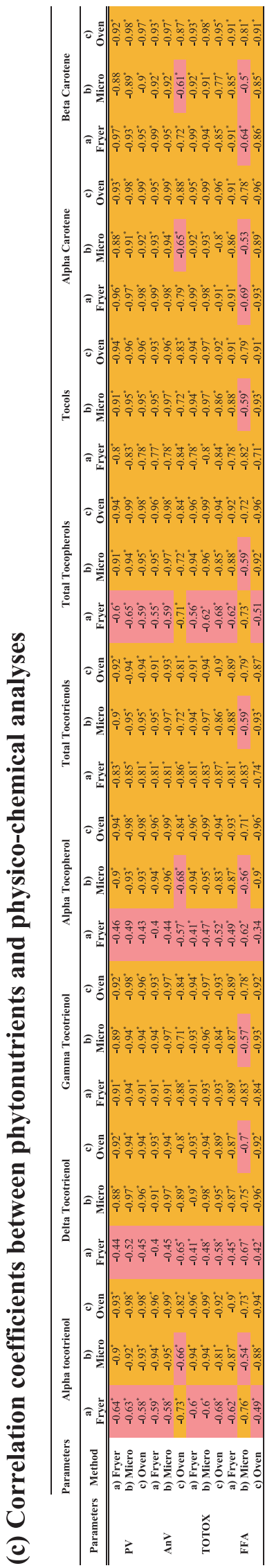


R. Loganathan, A. H. Ahmad Tarmizi, S. R. Vethakkan et al.
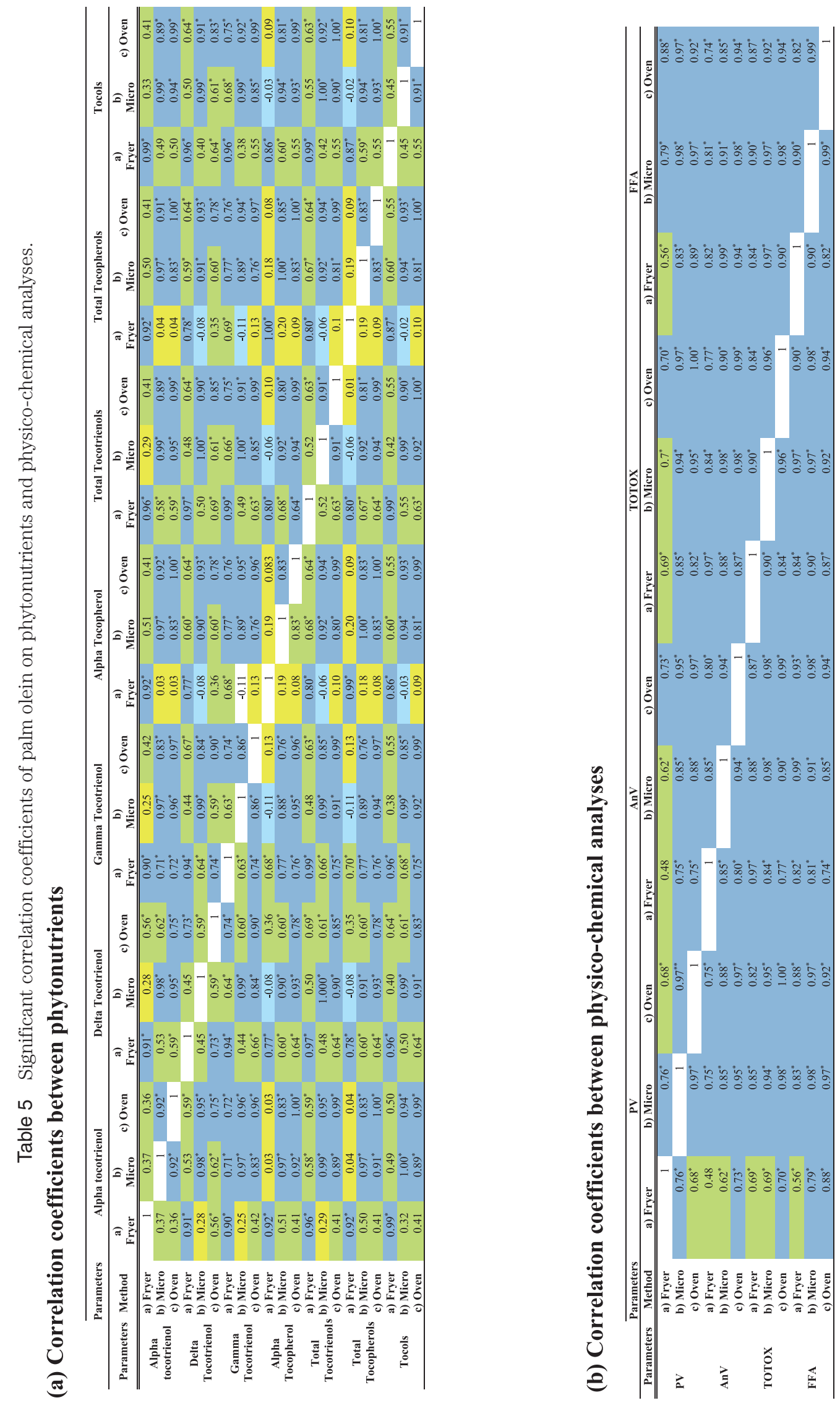


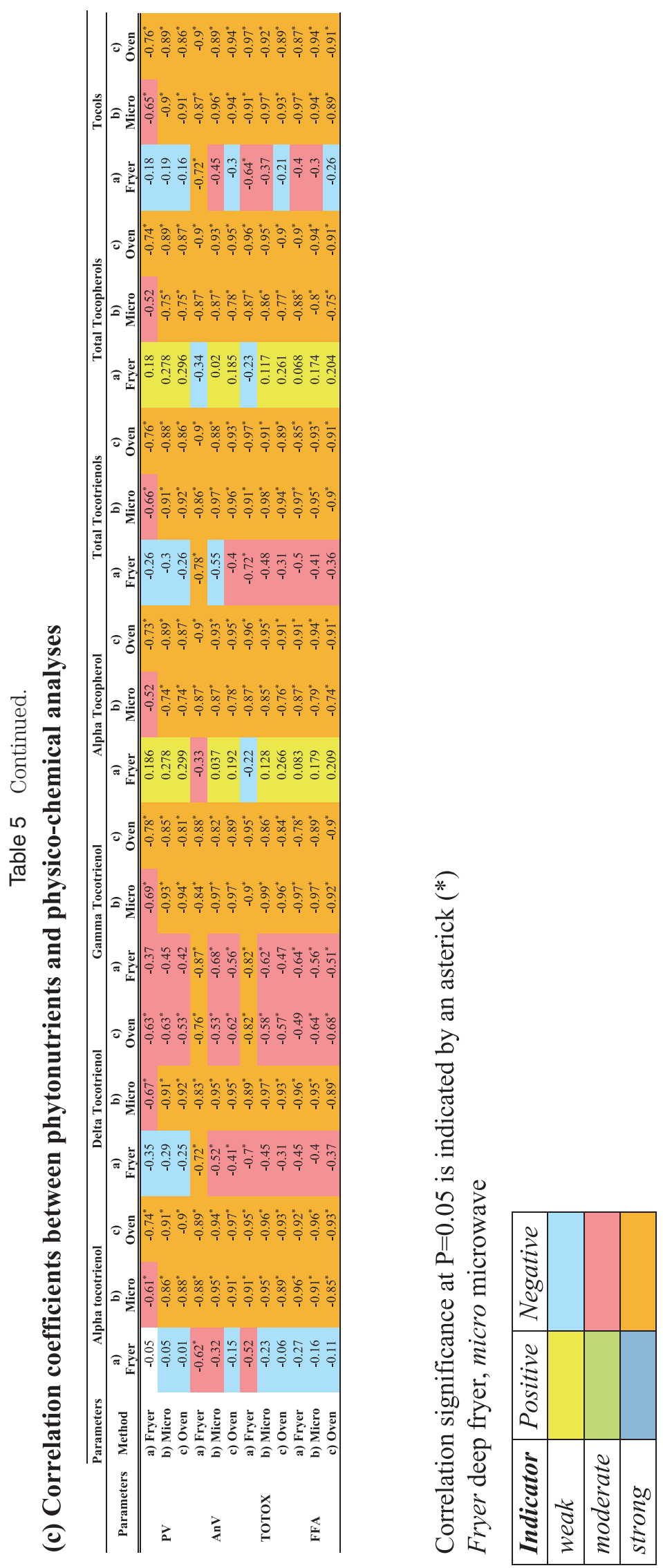




\section{Author Contributions}

R.L., K-T.T. and A.H.A.T. were involved in project conceptualization and design. R.L. and K-T.T. conducted the research and statistical analysis; prepared the manuscript and had primary responsibility for the content; and contributed for laboratory and data analyses. A.H.A.T. contributed to laboratory and data analyses, as well as manuscript editing. S.R.V edited the manuscript. All authors read and approved the manuscript.

\section{Funding}

This work was supported by research grants from the Malaysian Palm Oil Board (Project ID: PD16412).

\section{Declaration of Interest}

The authors have no relevant interests to declare.

\section{References}

1) Ahmad Tarmizi, A.H.; Ismail, R. Use of pilot plant scale continuous fryer to simulate industrial production of potato chips: thermal properties of palm olein blends under continuous frying conditions. Food Sci. Nutr. 2, 28-38 (2014).

2) Kushairi, A.; Singh, R.; Ong-Abdullah, M. The oil palm industry in Malaysia: Thriving with transformative technologies. J. Oil Palm Res. 29, 431-439 (2017).

3) Seppanen, C.M.; Song, Q.; Saari Csallany, A. The antioxidant functions of tocopherol and tocotrienol homologues in oils, fats, and food systems. J. Am. Oil Chem. Soc. 87, 469-481 (2010).

4) Chen, W.-A.; Chiu, C.P.; Cheng, W.-C.; Hsu, C.-K.; Kuo, M.-I. Total polar compounds and acid values of repeatedly used frying oils measured by standard and rapid methods. J. Food Drug Anal. 21, 58-65 (2013).

5) Schroeder, M.T.; Becker, E.M.; Skibsted, L.H. Molecular mechanism of antioxidant synergism of tocotrienols and carotenoids in palm oil. J. Agric. Food Chem. 54, 3445-3453 (2006).

6) Ahmad Tarmizi, A.H.; Lin, S.W. Quality assessment of palm products upon prolonged heat treatment. J. Oleo Sci. 57, 639-648(2008).

7) Loganathan, R.; Subramaniam, K.M.; Radhakrishnan, A.K.; Choo, Y.-M.; Teng, K.-T. Health-promoting effects of red palm oil: evidence from animal and human studies. Nutr. Rev. 75, 98-113(2017).

8) Ajose, O.A.; Adelekan, D.A.; Ajewole, E.O. Vitamin A status of pregnant Nigerian women: relationship to dietary habits and morbidity. Nutr. Health 17, 325-333
(2004).

9) Loganathan, R.; Vethakkan, S.R.; Radhakrishnan, A.K.; Razak, G.A.; Kim-Tiu, T. Red palm olein supplementation on cytokines, endothelial function and lipid profile in centrally overweight individuals: a randomised controlled trial. Eur. J. Clin. Nutr. 73, 609-616(2018).

10) AOCS. AOCS Official Method Cd 8b-90: Peroxide value, acetic acid, isooctane method. in Official Methods and Recommended Practices of the AOCS. American Oil Chemists Society Press, Champaign (2011).

11) AOCS. AOCS Official Method Cd 18-90: p-Anisidine value. in Official Methods and Recommended Practices of the AOCS. American Oil Chemists Society Press, Champaign (2011).

12) AOCS. AOCS Official Method Ce 1a-13: Determination of fatty acids in edible oils and fats by capillary GLC. in Official Methods and Recommended Practices of the AOCS. American Oil Chemists Society Press, Champaign (2013).

13) Sepulveda, D.; Barbosa-Canovas, G. Heat transfer in food products. in Transport phenomena in food processing. CRC Press, Boca Raton(2002).

14) Malheiro, R.; Oliveira, I.; Vilas-Boas, M.; Falcão, S.; Bento, A.; Pereira, J.A. Effect of microwave heating with different exposure times on physical and chemical parameters of olive oil. Food Chem. Toxicol. 47, 92-97(2009).

15) Yoshida, Y.; Niki, E.; Noguchi, N. Comparative study on the action of tocopherols and tocotrienols as antioxidant: chemical and physical effects. Chem. Phys. Lipids 123, 63-75 (2003).

16) Ahsan, H.; Ahad, A.; Siddiqui, W.A. A review of characterization of tocotrienols from plant oils and foods. $J$. Chem. Biol. 8(2), 45-59(2015).

17) Choe, E.; Min, D.B. Mechanisms and factors for edible oil oxidation. Compr. Rev. Food Sci. F. 5, 169-186 (2006).

18) Gupta, M.K. 4.1 Frying oils. in Bailey's Industrial Oil and Fat Products. 6th ed. Wiley (2005).

19) $\mathrm{Hu}, \mathrm{M}$; J Jacobsen, C. Oxidative stability and shelf life of foods containing oils and fats. Elsevier (2016).

20) Decker, E.; Elias, R.; McClements, D.J. Oxidation in foods and beverages and antioxidant applications: management in different industry sectors. Elsevier (2010).

21) Cheikhousman, R.; Zude, M.; Bouveresse, D.J.-R.; Léger, C.L.; Rutledge, D.N.; Birlouez-Aragon, I. Fluorescence spectroscopy for monitoring deterioration of extra virgin olive oil during heating. Anal. Bioanal. Chem. 382, 1438-1443(2005).

22) Daskalaki, D.; Kefi, G.; Kotsiou, K.; Tasioula-Margari, M. Evaluation of phenolic compounds degradation in virgin olive oil during storage and heating. J. Food Nutr. Res. 48, 31-41 (2009). 
23) Yahyaoui, A.; Rigane, G.; Salem, R.B. Microwave heating of different commercial Tunisian olive oil: Regarding to exposure times on physical and chemical parameters properties. Biochem. Physiol. 3, 131 (2014).

24） Kreps, F.; Vrbiková, L.; Schmidt, Š.; Sekretár, S.; Híreš, O. Chemical changes in microwave heated vegetable oils. Eur. J. Lipid Sci. Technol. 116, 1685-1693 (2014).

25） Dostalova, J.; Hanzlik, P.; Reblova, Z.; Pokorny, J.; Sakurai, H. Oxidative changes of vegetable oils during microwave heating. Czech. J. Food Sci. 23(6), 230-239 (2005).

26) Poiana, M.A. Enhancing oxidative stability of sunflower oil during convective and microwave heating using grape seed extract. Int. J. Mol. Sci. 13, 9240-9259 (2012).

27) Ali, M.A.; Nouruddeen, Z.B.; Muhamad, I.I.; Latip, R.A.; Othman, N.H. Effect of microwave heating on the quality characteristics of canola oil in presence of palm olein. Acta Sci. Pol. Technol. Aliment. 12, 241-251 (2013).

28) Mahmoud, E.A.E.-M.; Dostálová, J.; Pokorny̆, J.; Lukešová, D.; Doležal, M. Oxidation of olive oils during microwave and conventional heating for fast food preparation. Czech. J. Food Sci. 27, S173-S177 (2009).

29) Caponio, F.; Pasqualone, A.; Gomes, T. Changes in the fatty acid composition of vegetable oils in model doughs submitted to conventional or microwave heating. Int. J. Food Sci. Technol. 38, 481-486 (2003).

30) Pristouri, G.; Badeka, A.; Kontominas, M. Effect of packaging material headspace, oxygen and light transmission, temperature and storage time on quality characteristics of extra virgin olive oil. Food Control. 21, 412-418(2010).

31) SIRIM. Malaysian Standard for Palm Oil Specifications. MS 814:2007 Department of Standards Malaysia (2007).

32) Ahmad Tarmizi, A.H.; Ismail, R.; Kuntom, A. Effect of frying on the palm oil quality attributes-A review. $J$. Oil Palm Res. 28, 145-153 (2016).

33) Mishra, R.; Sharma, H.K.; Sarkar, B.C.; Singh, C. Thermal oxidation of rice bran oil during oven test and mi- crowave heating. J. Food Sci. Technol. 49, 221-227 (2012).

34) Li, X.; Bremer, G.C.; Connell, K.N.; Ngai, C.; Pham, Q.A.T.; Wang, S. et al. Changes in chemical compositions of olive oil under different heating temperatures similar to home cooking. J. Food Chem. Nutr. 4, 7-15 (2016).

35) Casal, S.; Malheiro, R.; Sendas, A.; Oliveira, B.P.; Pereira, J.A. Olive oil stability under deep-frying conditions. Food Chem. Toxicol. 48, 2972-2979 (2010).

36) Aladedunye, F.; Przybylski, R. Performance of palm olein and modified rapeseed, sunflower, and soybean oils in intermittent deep-frying. Eur. J. Lipid Sci. Technol. 116, 144-152 (2014).

37) Serjouie, A.; Tan, C.P.; Mirhosseini, H.; Che Man, Y. Effect of vegetable-based oil blends on physicochemical properties of oils during deep-fat frying. Am. J. Food Technol. 5, 310-323 (2010).

38) Fan, H.; Sharifudin, M.; Hasmadi, M.; Chew, H. Frying stability of rice bran oil and palm olein. Int. Food Res. J. 20, 403 (2013).

39) Andrikopoulos, N.K.; Kalogeropoulos, N.; Falirea, A.; Barbagianni, M.N. Performance of virgin olive oil and vegetable shortening during domestic deep-frying and pan-frying of potatoes. Int. J. Food Sci. Technol. 37, 177-190 (2002).

40) Uslu, N.; Özcan, M.M. Determination of the physicochemical changes in the different vegetable oils after fat-product interaction during frying process. Iran. $J$. Chem. Chem. Eng. 37 (6), 191-199(2018).

41) Ali, M.A.; Nouruddeen, Z.B.; Muhamad, II; Latip, R.A.; Othman, N.H. Effect of microwave heating on the quality characteristics of canola oil in presence of palm olein. Acta Sci. Pol. Technol. Aliment 12, 241-252 (2013).

42) Poiana, M.-A. Enhancing oxidative stability of sunflower oil during convective and microwave heating using grape seed extract. Int. J. Mol. Sci. 13, 9240-9259 (2012).

43) Hornero-Méndez, D.; Pérez-Gálvez, A.; Mínguez-Mosquera, M.I. A rapid spectrophotometric method for the determination of peroxide value in food lipids with high carotenoid content. J. Am. Oil Chem. Soc. 78, 1151-1155(2001). 